NBER WORKING PAPER SERIES

\title{
FIPIT: A SIMPLE, FAST GLOBAL METHOD FOR SOLVING MODELS WITH TWO ENDOGENOUS STATES \& OCCASIONALLY BINDING CONSTRAINTS
}

\author{
Enrique G. Mendoza \\ Sergio Villalvazo \\ Working Paper 26310 \\ http://www.nber.org/papers/w26310 \\ NATIONAL BUREAU OF ECONOMIC RESEARCH \\ 1050 Massachusetts Avenue \\ Cambridge, MA 02138 \\ September 2019
}

We thank Javier Bianchi, Pablo D'Erasmo, Bora Durdu, Vincenzo Quadrini and Urban Jermann for helpful comments and suggestions. The views expressed herein are those of the authors and do not necessarily reflect the views of the National Bureau of Economic Research.

NBER working papers are circulated for discussion and comment purposes. They have not been peer-reviewed or been subject to the review by the NBER Board of Directors that accompanies official NBER publications.

(C) 2019 by Enrique G. Mendoza and Sergio Villalvazo. All rights reserved. Short sections of text, not to exceed two paragraphs, may be quoted without explicit permission provided that full credit, including $\odot$ notice, is given to the source. 
FiPIt: A Simple, Fast Global Method for Solving Models with Two Endogenous States \&

Occasionally Binding Constraints

Enrique G. Mendoza and Sergio Villalvazo

NBER Working Paper No. 26310

September 2019

JEL No. E17,E44,F34,F41

\begin{abstract}
We propose a simple and fast fixed-point iteration algorithm FiPIt to obtain the global, non-linear solution of macro models with two endogenous state variables and occasionally binding constraints. This method uses fixed-point iteration on Euler equations to avoid solving two simultaneous nonlinear equations (as with the time iteration method) or creating modified state variables requiring irregular interpolation (as with the endogenous grids method). In the smallopen-economy RBC and Sudden Stops models provided as examples, FiPIt is used on the bonds and capital Euler equations to solve for the bonds decision rule and the capital pricing function. In a standard Matlab platform, FiPIt solves both models much faster than time iteration and various hybrid methods. The choice of functions that FiPIt iterates on using the Euler equations can vary across models, and there can be more that one arrangement for the same model.
\end{abstract}

\author{
Enrique G. Mendoza \\ Department of Economics \\ University of Pennsylvania \\ 3718 Locust Walk \\ Philadelphia, PA 19104 \\ and NBER \\ egme@sas.upenn.edu \\ Sergio Villalvazo \\ Department of Economics \\ University of Pennsylvania \\ The Ronald O. Perelman Center \\ for Political Science and Economics \\ 133 South 36th Street \\ Philadelphia, PA 19104 \\ vsergio@sas.upenn.edu
}

A data appendix is available at http://www.nber.org/data-appendix/w26310 


\section{Introduction}

Important branches of the recent macroeconomics literature study quantitative solutions of models in which constraints are triggered endogenously (i.e. they are "occasionally binding"), as in studies of the zero-lower-bound on interest rates or financial crises triggered by credit constraints. Because these models typically feature non-linear decision rules that lack analytic solutions and capture precautionary saving behavior, global solution methods (e.g. time iteration or endogenous grids methods) are the preferable tool for solving them. Global methods are, however, less practical than perturbation methods, because of limitations that make them slow and difficult to implement with widely used software (e.g. Matlab). On the other hand, perturbation methods for solving models with occassionally binding constraints have caveats that limit the scope of the findings that can be derived from using them (see Aruoba et al. [2006], Durdu et al. [2019]). In particular, in the OccBin toolbox developed by Guerrieri and Iacoviello [2015] the constraint becomes binding as an exogenous surprise or "MIT shock". If the constraint does not bind at steady state, decision rules when the constraint is not binding match those of a model without the constraint and expectations that the constraint may bind in the future are irrelevant. OccBin decision rules also abstract from precautionary savings and disregard the risk effects of the occassionally binding constraint. In contrast, these are important elements driving macro dynamics in global solutions and they play a central role in quantitative models examining the implications of financial frictions (e.g. Bianchi and Mendoza [2018], Mendoza [2010]).

This paper proposes a simple and fast algorithm to obtain the global solution of models with two endogenous states and occasionally binding constraints. This algorithm is denoted as FiPIt because it is based on the well-known fixed-point iteration approach to solve systems of transcendental equations. It is easy to implement in a Matlab platform and is significantly faster than the standard time iteration algorithm and several hybrid alternatives. FiPIt's solution strategy builds on the class of time iteration methods that originated in the work of Coleman [1990], who first proposed a global solution method based on policy function iterations of the Euler equation. Since then, various enrichments and modifications of this approach have been developed, in particular the endogenous grids method proposed by Carroll [2006] (see Rendahl [2015] for a general discussion of these methods and an analysis of their convergence properties). FiPIt differs from these methods in that it applies the fixed-point iteration method to solve a model's Euler equations. For instance, in the small-open-economy RBC and Sudden Stops models provided as examples in this paper, the 
bonds (capital) Euler equation is used to solve directly for a "new" bonds decision rule (capital pricing function) without the need of a nonlinear solver. The capital decision rule is solved for in "exact" form using the models' optimality conditions.

The endogenous grids method also avoids using a nonlinear solver, but it does so by defining alternative state variables so that obtaining analytic solutions of Euler equations for control variables (e.g. consumption, investment) requires irregular interpolation of functions defined over endogenous grids of the original state space. This is innocuous in one-dimensional problems, but in two- and higher-dimensional problems it requires elaborate interpolation methods to tackle the non-rectangular nature of the endogenous grids. In particular, Ludwig and Schön [2018] developed a method using Delaunay interpolation, and showed that it is significantly faster that standard time iteration. ${ }^{1}$ Alternatively, Brumm and Grill [2014] proposed a a variant of the time iteration method that still uses a non-linear solver but gains speed and accuracy by updating grid nodes to track decision rule kinks using also Delaunay interpolation. In contrast, FiPIt retains the original state variables so that standard multi-linear interpolation on regular grids can be used.

We apply the algorithm to solve the models examined by Mendoza [2010], which include RBC and Sudden Stops (SS) models of a small open economy. The SS model includes an occasionally binding credit constraint limiting intertemporal debt and working capital not to exceed a fraction of the market value of physical capital (i.e. pledgeable collateral). The results show that, relative to the time iteration method, FiPIt reduces execution time by factors of 18.1 for the RBC model and 2.5 for the SS model. ${ }^{2}$ Ludwig and Schön [2018] report reductions by factors of 2.7 to 4.1 using endogenous grids with Delaunay interpolation v. standard time iteration, or 1.8 to 2.5 using their hybrid method v. standard time iteration, when solving a perfect-foresight model of human capital accumulation in a small open economy. ${ }^{3}$ We also found that FiPIt continues to perform well for several parameter variations, despite the well-known drawback of fixed-point iteration methods indicating that their convergence is not guaranteed. Execution times for six parameter variations

\footnotetext{
${ }^{1}$ Adjacent points in the endogenous grids do not generally match adjacent nodes in the matrix formed by the original grids. Ludwig and Schon tackled this problem using Delaunay interpolation. They also proposed a hybrid method that uses an exogenous grid for one of the endogenous states and an endogenous grid for the second.

${ }^{2}$ We used Matlab version R2017a on a Windows 10 laptop with an Intel Core i7-6700HQ 2.60GHz chip, 4 physical cores and $16 \mathrm{~GB}$ of RAM. The state space for the RBC (SS) model has 80 (72) nodes on foreign assets and 30 on domestic capital. The RBC (SS) model solved in 100 (810) seconds, compared with 1,808 (1,986) using the time iteration method.

${ }^{3}$ They report faster solution times for each individual scenario than with our algorithm but these are not comparable due to differences in models and hardware. We solve a stochastic model with three shocks, capital accumulation and adjustment costs, and a credit constraint that depends on the model's two endogenous states and a market price. They solve a deterministic model in which human capital is an accumulable factor produced with an exponential technology and a no-borrowing constraint. We do not have details about the software and hardware they used.
} 
of the SS model were smaller than using time iteration by factors of 2.0 to 6.0.

In addition to the Delaunay interpolation, a second drawback of the endogenous grids method relative to the FiPIt method is that it still requires a root-finder in order to determine equilibrium solutions in points of the state space in which occasionally binding constraints bind (see Ludwig and Schön [2018]). FiPIt requires a non-linear solver only if the solution of the allocations when the constraint binds cannot be separated from the solution of the multiplier of the constraint. The two are separable in models that feature several widely-used occasionally binding constraints, including standard no-borrowing constraints, maximum debt limits, and constraints on debt-to-income and loan-to-value ratios that depend on endogenous variables. Solving variations of the SS model using these constraints, FiPIt reduced execution time relative to the time iteration method by a factor of 13.0 for a loan-to-value-ratio constraint and 17.9 for a maximum debt limit.

There are applications in the literature that solve models using fixed-point iteration algorithms with some features similar to the one we proposed here. Boz and Mendoza [2014] solved an openeconomy model with an occasionally binding credit constraint and Bayesian learning. Bianchi and Mendoza [2018] and Bianchi et al. [2016] solved models with collateral constraints iterating on pricing functions when evaluating collateral. Carroll [2011] described and implemented a fixed-point iteration algorithm for solving the workhorse complete-markets RBC model of a closed economy. This paper differs significantly from these studies in that we develop an algorithm that solves models with two endogenous states, and it does so easily and fast in a standard Matlab platform. FiPIt can be used in variety of models with two endogenous states. The choice of functions that are iterated on using the Euler equations can vary across models, and there can be more that one arrangement for the same model.

The rest of the paper proceeds as follows. The next Section describes the principles of the algorithm in the simple case of a model of savings with endowment income, and uses this example also to explain how FiPIt differs from the time iteration and endogenous grids methods. Section 3 describes the RBC and SS models and provides a step-by-step description of the complete algorithm. Section 4 examines quantitative results for both models, evaluates the robustness of the algorithm, and conducts performance comparisons with alternative algorithms, including the standard time iteration method. Section 5 presents conclusions. In addition, the Matlab codes are available online, together with an online Appendix that provides a user's guide to the codes. 


\section{A Fixed-Point Iteration Algorithm for a Simple Savings Model}

We describe the principles of the FiPIt method using a savings model with stochastic endowment income and an exogenous interest rate. This model is a workhorse of various branches of the macro literature, including consumption and savings in partial equilibrium, heterogeneous agents models with incomplete markets, and international macro models of the small open economy.

A representative agent chooses consumption and savings plans so as to maximize a standard expected utility function:

$$
E_{0}\left\{\sum_{t=0}^{\infty} \beta^{t} u\left(c_{t}\right)\right\} .
$$

subject to the budget or resource constraint:

$$
c_{t}=e^{z_{t}} \bar{y}+b_{t}-q b_{t+1} .
$$

and a debt limit:

$$
b_{t+1} \geq-\varphi
$$

In the utility function, $\beta \in(0,1)$ is the subjective discount factor and $u(\cdot)$ is the period utility function, which can be any standard twice, continuously differentiable and concave utility function, although the CRRA functional form is the one used most often:

$$
u\left(c_{t}\right)=\frac{c_{t}^{1-\sigma}}{1-\sigma}
$$

where $\sigma$ is the relative risk aversion coefficient. In the resource constraint, $e^{z_{t}} \bar{y}$ is stochastic income with mean $\bar{y}$ and shocks $z_{t}$ of exponential support $e^{z_{t}}, b_{t}$ are holdings of one-period, non-statecontingent discount bonds traded in a frictionless credit market. In a partial equilibrium model of savings or a model of a small open economy, the real interest rate $r$ is exogenous, so the price of

bonds is also exogenous and given by $q \equiv \frac{1}{1+r}$. In a general equilibrium model of heterogeneous agents, the above optimization problem is solved by each individual agent facing idiosyncratic income uncertainty, and the interest rate is endogenously determined so as to clear the bond market. The FiPIt method can be used in all of these models, except that in the heterogeneous agents model we would also need to iterate on the interest rate until the bond market clears. We focus on the small open economy case to simplify the exposition.

If the utility function satisfies the Inada condition and income shocks follow a discrete Markov 
process or a truncated continuous distribution, the debt limit follows from Aiyagari's Natural Debt Limit: agent's never choose optimal plans that leave them exposed to the risk of non-positive consumption, and hence never borrow more than the annuity value of the lowest income realization. Alternatively, agents may face an ad-hoc debt limit tighter than the natural debt limit. Thus, the model includes an occasionally binding constraint, albeit of a simple form: $b_{t+1} \geq-\varphi$.

The Euler equation for bond holdings is

$$
u_{c}(t)=(1+r) \beta E_{t}\left[u_{c}(t+1)\right]+\mu_{t},
$$

where $u_{c}(t)$ is the marginal utility of $c_{t}$ and $\mu_{t}$ is the multiplier on the debt limit. Note that using the resource constraint to substitute for consumption, the Euler equation can be expressed as:

$$
u_{c}\left(e^{z_{t}} \bar{y}+b_{t}-q b_{t+1}\right)=(1+r) \beta E_{t}\left[u_{c}\left(e^{z_{t+1}} \bar{y}+b_{t+1}-q b_{t+2}\right)\right]+\mu_{t}
$$

A competitive equilibrium for this economy is defined by stochastic sequences $\left[c_{t}, b_{t+1}\right]_{t=0}^{\infty}$ that satisfy equations (3) and (4) for all $t$. The economy has a well-defined limiting distribution of $(b, y)$ (i.e. a stochastic steady state) only if $\beta(1+r)<1$ (see Ljungqvist and Sargent [2012], Ch. 18). This condition is also a general equilibrium outcome in heterogeneous agents models, because otherwise all agents would want an infinite amount of bonds, which is inconsistent with market clearing in the market of risk-free bonds.

Since there are no inefficiencies affecting the small open economy (other than the incompleteness of asset markets), the competitive equilibrium can be represented as the solution to the following dynamic programming problem:

$$
V(b, z)=\max _{c, b^{\prime}}\left\{\frac{c^{1-\sigma}}{1-\sigma}+\beta \sum_{z^{\prime}} \pi\left(z^{\prime}, z\right) V\left(b^{\prime}, z^{\prime}\right)\right\}
$$

subject to

$$
\begin{gathered}
c=e^{z} \bar{y}+b-q b^{\prime} \\
b^{\prime} \geq-\varphi
\end{gathered}
$$

The solution to the above Bellman equation is characterized by a decision rule $b^{\prime}(b, z)$ and the associated value function $V(b, z)$, and the decision rule together with the Markov process of the 
shocks induce a joint ergodic (unconditional) distribution of bonds and income $\lambda(b, z)$.

"Euler equation" methods typically solve for $b^{\prime}(b, z)$ over a discrete state space of $(b, z)$ pairs using the recursive equilibrium conditions that follow from the first-order-conditions of the above Bellman equation:

$$
\begin{gathered}
c(b, z)^{-\sigma} \geq \beta R \sum_{z^{\prime}} \pi\left(z^{\prime}, z\right)\left[\left(c\left(b^{\prime}(b, z), z^{\prime}\right)\right)^{-\sigma}\right] \\
c(b, z)=e^{z} \bar{y}+b-q b^{\prime}(b, z) .
\end{gathered}
$$

The recursive equilibrium of the model is then defined as the pair of decision rules $c(b, z), b^{\prime}(b, z)$ that satisfy these two conditions.

The FiPIt method poses a conjecture of the decision rule $\hat{b}_{j}^{\prime}(b, z)$ in iteration $j$, and generates its associated consumption function as $c_{j}(b, z)=e^{z} \bar{y}+b-q \hat{b}_{j}^{\prime}(b, z)$. Then, it combines the above first-order conditions to obtain this equation:

$$
c_{j+1}(b, z)=\left\{\beta R \sum_{z^{\prime}} \pi\left(z^{\prime}, z\right)\left[\left(c_{j}\left(\hat{b}_{j}^{\prime}(b, z), z^{\prime}\right)\right)^{-\sigma}\right]\right\}^{-\frac{1}{\sigma}}
$$

In the right-hand-side of this Euler equation, we need the value of $c_{t+1}$, which is obtained by evaluating the consumption function at the $\mathrm{t}+1$-values of the state variables: $\left.c_{j}\left(\hat{b}_{j}^{\prime}(b, z), z^{\prime}\right)\right)$. Since $\hat{b}_{j}^{\prime}(b, z)$ is defined only on the nodes of the grid of bonds, this consumption function is interpolated over its first argument in order to determine $c_{j}\left(\hat{b}_{j}^{\prime}(b, z), z^{\prime}\right)$ (i.e. the value of $c_{t+1}$ implied by the conjectured consumption function). Once this is done, the Euler equation solves directly for a new consumption function $c_{j+1}(b, z)$ without a non-linear solver. Using the resource constraint, this new consumption function yields a new decision rule for bonds $b_{j+1}^{\prime}(b, z)$, which is re-set to $b_{j+1}^{\prime}(b, z)=-\varphi$ if $b_{j+1}^{\prime}(b, z) \leq-\varphi$. Then the decision rule conjecture is updated to $\hat{b}_{j+1}^{\prime}(b, z)$ as a convex combination of $\hat{b}_{j}^{\prime}(b, z)$ and $b_{j+1}^{\prime}(b, z): \hat{b}_{j+1}^{\prime}(b, z)=(1-\rho) \hat{b}_{j}^{\prime}(b, z)+\rho b_{j+1}^{\prime}(b, z)$. The process is repeated until $b_{j+1}^{\prime}(b, z)=\hat{b}_{j}^{\prime}(b, z)$ for all $(b, z)$, up to a convergence criterion.

Three points raised by Judd [1998] about fixed-point iteration algorithms like this one are worth recalling. First, using colocation methods instead of solving for a finite state space, the fixedpoint iteration method can be represented in a form analogous to the Parameterized Expectations method, because the latter is a fixed-point iteration method that uses simulation and regression to construct conditional expectations. Second, using $0<\rho<1(\rho>1)$ to set the decision rule of the next iteration is useful to address possible instability (slow convergence) of the algorithm. Third, a finite state space may be preferable to colocation methods to define the decision rules depending 
on whether we expect decision rules to be smooth or to have strong curvature. The latter can be particularly important in models with occasionally binding constraints that depend on endogenous variables, such as credit constraints that depend on collateral prices and yield U-shaped decision rules because of the Fisherian debt-deflation mechanism (see Bianchi and Mendoza [2018]). This will be the case in the SS model solved in the next Section.

Fixed-point iteration differs from the time iteration method because the latter applies the conjectured decision rule $\hat{b}_{j}^{\prime}(b, z)$ only to substitute for the term $b_{t+2}$ in the right-hand-side of the Euler equation (5), and then uses a non-linear solver to solve the resulting non-linear equation for the optimal choice of $b_{t+1}$ as a function of $\left(z_{t}, b_{t}\right)$. Hence, we can think of the fixed-point iteration method as a "proxy time iteration method" that substitutes for the $b_{t+1}$ in the right-hand-side of the Euler equation with a proxy that is defined to be the conjectured decision rule, instead of treating that $b_{t+1}$ term as endogenous. Fixed-point iteration is also different from the endogenous grids method, because it does not redefine the endogenous state variable and instead solves the problem over the original rectilinear grids $(b, z)$. Still, fixed-point iteration retains the main computational advantage of the endogenous grids method, which is that the Euler equation is reduced to an equation with an analytic solution for the decision rule, avoiding the need to use non-linear solvers.

By not proceeding as the time iteration or endogenous grids methods, however, the fixed-point iteration method seems to solve the "incorrect" Euler equation. Yet, as we document later in the paper, the solutions satisfy the same equilibrium conditions and are negligibly different from those obtained using standard time iteration. This is because the algorithm is simply an application of the standard fixed-point iteration approach to solve transcendental equations.

\section{The FiPIt Method for Two-Dimensional Models}

This Section provides a detailed description of the steps that the FiPIt method follows to solve a model with two endogenous states and an occasionally binding constraint. The model pertains to a small open economy with two endogenous states, capital $(k)$ and net foreign assets $(b)$, and a credit constraint. If the constraint never binds, the algorithm solves a standard RBC model of a small open economy, and if it binds it solves a model with endogenous financial crises or Sudden Stops. 


\subsection{Model structure and equilibrium conditions}

The model is the same as in Mendoza [2010], except that the preferences with endogenous discounting are replaced with standard time-separable expected utility with exogenous discounting at rate $\beta$. The economy is inhabited by a representative firm-household with preferences defined over stochastic sequences of consumption $c_{t}$ and labor supply $L_{t}$, for $t=0, \ldots, \infty$, given by:

$$
E_{0}\left[\sum_{t=0}^{\infty} \beta^{t} \frac{\left(c_{t}-\frac{L_{t}^{\omega}}{\omega}\right)^{1-\sigma}}{1-\sigma}\right]
$$

The agent chooses sequences of consumption, labor, investment, and holdings of real, one-period international bonds, $b_{t+1}$ (the agent borrows when $b_{t+1}<0$ ), so as to maximize the above utility function subject to the following budget and collateral constraints:

$$
\begin{gathered}
c_{t}(1+\tau)+k_{t+1}-(1-\delta) k_{t}+\frac{a}{2} \frac{\left(k_{t+1}-k_{t}\right)^{2}}{k_{t}}=A_{t} F\left(k_{t}, L_{t}, v_{t}\right)-p_{t} v_{t}-\phi\left(R_{t}-1\right)\left(w_{t} L_{t}+p_{t} v_{t}\right)-q_{t}^{b} b_{t+1}+b_{t} \\
q_{t}^{b} b_{t+1}-\phi R_{t}\left(w_{t} L_{t}+p_{t} v_{t}\right) \geq-\kappa q_{t} k_{t+1}
\end{gathered}
$$

The right-hand-side of the budget constraint is the sum of net profits from production and the resources generated by trading assets abroad. Net profits are equal to gross production minus the cost of imported inputs minus the servicing of foreign working capital loans for labor and imported inputs. Gross output is represented by a constant-returns-to-scale technology, $A_{t} F\left(k_{t}, L_{t}, v_{t}\right)=$ $\exp \left(\epsilon_{t}^{A}\right) A k_{t}^{\gamma} L_{t}^{\alpha} v_{t}^{\eta}$, that requires capital, $k_{t}$, labor and imported inputs, $v_{t}$, to produce a tradable good sold at a world-determined price (normalized to unity without loss of generality). TFP is subject to a random shock $\epsilon_{t}^{A}$ with exponential support around a mean of $A$. Working capital loans pay for a fraction $\phi$ of the cost of imported inputs and labor in advance of sales. These loans are obtained from foreign lenders at the beginning of each period and repaid at the end. Lenders charge the world gross real interest rate $R_{t}=R \exp \left(\epsilon_{t}^{R}\right)$ on these loans, where $\epsilon_{t}^{R}$ is an interest rate shock around a mean value $R$. Imported inputs are purchased at an exogenous relative price in terms of the world's numeraire $p_{t}=p \exp \left(\epsilon_{t}^{P}\right)$, where $p$ is the mean price and $\epsilon_{t}^{P}$ is a shock to the world price of imported inputs (i.e., a terms-of-trade shock). The shocks $\epsilon_{t}^{A}, \epsilon_{t}^{R}$, and $\epsilon_{t}^{P}$ follow a joint first-order Markov process. The resources generated by trading assets abroad are given by $-q_{t}^{b} b_{t+1}+b_{t}$, where $q_{t}$ is the price of the international bonds, which satisfies $q_{t}^{b}=R_{t}^{-1}$.

The left-hand-side of the budget constraint is the sum of consumption expenditures, investment 
and capital adjustment costs. Gross investment is $i_{t}=k_{t+1}-(1-\delta) k_{t}$ and gross investment inclusive of adjustment costs is $\tilde{i}_{t}=k_{t+1}-(1-\delta) k_{t}+\frac{a}{2} \frac{\left(k_{t+1}-k_{t}\right)^{2}}{k_{t}}$. Since government expenditures are not included in the model, we include a time-invariant consumption $\operatorname{tax} \tau$ that is used to calibrate the model to match the average share of government expenditures in GDP in the data. This is done so that consumption and investment shares in the model can match their data counterparts. Since the tax is constant it does not distort the savings-consumption margin and it does not create a time-varying distortion on labor supply.

The credit constraint limits the total debt, which is equal to intertemporal debt plus working capital financing, not to exceed the fraction $\kappa$ of the market value of the end-of-period capital stock. This is a more complex constraint than borrowing constraints of the class $b_{t+1} \geq-\varphi$, widely used in heterogeneous agents models and also in the algorithm proposed by Ludwig and Schön [2018]. Notice that the prices $q_{t}$ and $w_{t}$ that appear in this constraint (and the wage in the budget constraint), are endogenous market prices taken as given by the agent when solving its optimization problem. As in Mendoza [2010], the wage rate must be on the labor supply curve (i.e. it must equal the tax-adjusted marginal disutility of labor), which requires $w_{t}=L^{\omega-1}(1+\tau)$, and the price of capital must satisfy the optimality condition requiring that $q_{t}=\frac{\partial \tilde{i}_{t}}{\partial k_{t+1}}$. With these simplifications noted, the competitive equilibrium of the economy can be represented with the optimization problem of the firm-household, instead of defining separate problems for households and firms. This equilibrium, however, cannot be represented as the solution to a planner's problem represented by a single Bellman equation, because the planner would internalize the responses of wages and asset prices to its optimal plans, while the representative firm-household does not.

Defining $\lambda_{t}$ and $\mu_{t}$ as the future-value multipliers of the budget and collateral constraints respectively, the model's equilibrium conditions in sequential form are:

$$
\begin{gathered}
\left(c_{t}-\frac{L_{t}^{\omega}}{\omega}\right)^{-\sigma}=\lambda_{t}(1+\tau) \\
A_{t} F_{L_{t}}\left(k_{t}, L_{t}, v_{t}\right)=w_{t}\left(1+\phi\left(R_{t}-1\right)+\frac{\mu_{t}}{\lambda_{t}} \phi R_{t}\right) \\
A_{t} F_{v_{t}}\left(k_{t}, L_{t}, v_{t}\right)=p_{t}\left(1+\phi\left(R_{t}-1\right)+\frac{\mu_{t}}{\lambda_{t}} \phi R_{t}\right) \\
\lambda_{t}=\frac{1}{q_{t}^{b}} \beta E_{t}\left[\lambda_{t+1}\right]+\mu_{t}
\end{gathered}
$$




$$
\begin{gathered}
\lambda_{t}=\frac{1}{q_{t}} \beta E_{t}\left[\lambda_{t+1}\left(d_{t+1}+q_{t+1}\right)\right]+\mu_{t} \kappa \\
d_{t} \equiv \exp \left(\epsilon_{t}^{A}\right) F_{k_{t}}-\delta+\frac{a}{2} \frac{\left(k_{t+1}-k_{t}\right)^{2}}{k_{t}^{2}} \\
q_{t}=\frac{\partial \tilde{i}_{t}}{\partial k_{t+1}}=1+a\left(\frac{k_{t+1}-k_{t}}{k_{t}}\right) \\
w_{t}=L^{\omega-1}(1+\tau) \\
c_{t}(1+\tau)+k_{t+1}-(1-\delta) k_{t}+\frac{a}{2} \frac{\left(k_{t+1}-k_{t}\right)^{2}}{k_{t}}=A_{t} F\left(k_{t}, L_{t}, v_{t}\right)-p_{t} v_{t}-\phi\left(R_{t}-1\right)\left(L_{t}^{\omega}(1+\tau)+p_{t} v_{t}\right)-q_{t}^{b} b_{t+1}+b_{t}
\end{gathered}
$$

Solving this model with the time iteration method requires solving the Euler equations (16) and (17) as part of a system of non-linear equations. Given conjectures of the decision rules for capital and bonds, and simplifying using the other equilibrium conditions, the two Euler equations form a two-equation system that yields the "new" decision rules. When the collateral constraint does not bind, these two Euler equations have their standard forms. When the constraint binds, the multiplier $\mu_{t}$ is an additional endogenous variable and there is an additional equation, which is the constraint holding with equality. The solution can still be reduced to a two-equation system, by using the constraint to substitute for $q_{t}^{b} b_{t+1}$ together with the conjectured decision rules so as to obtain a two-equation system in $k_{t+1}$ and $\mu_{t} .{ }^{4}$

Solving with the endogenous grid method requires defining grids for two alternative state variables $\left(s^{1}, s^{2}\right)$ such that $s_{t}^{1} \equiv q_{t}^{b} b_{t+1}$ and $s_{t}^{2} \equiv k_{t+1} /(1-\delta)$, and then proceeding as in Ludwig and Schön [2018] to first determine the values of $\left(b_{t+1}, k_{t+1}\right)$ associated with each $\left(s_{t}^{1}, s_{t}^{2}\right)$ pair, then use the optimality conditions (including the Euler equations) to solve for the contemporaneous controls, particularly $\left(c_{t}, i_{t}\right)$, and then use the resource constraint and the definition of gross investment to extract the implied values of the original endogenous states $\left(b_{t}, k_{t}\right)$, namely the endogenous grids. When solving for the contemporaneous controls, the optimality conditions form a system of equations that can be solved for analytically, thus avoiding the need to use a non-linear solver, but the endogenous grids of $\left(b_{t+1}, k_{t+1}\right)$ are irregular, so interpolation of the relevant functions required to obtain the solution of the system is implemented using Delaunay interpolation. ${ }^{5}$ As noted earlier,

\footnotetext{
${ }^{4}$ If the solution implies a value of $b_{t+1}$ lower than the lower bound of the grid of bonds, we set $b_{t+1}$ to that lower bound and solve again the two-equation system for the values of $k_{t+1}$ and $\mu_{t+1}$ consistent with that value of $b_{t+1}$. Hence, the lower bound of the bonds grid is still treated as a constraint of the form $b_{t+1} \geq-\varphi$.

${ }^{5}$ The Ludwig-Schon algorithm still needs to solve a non-linear equation in order to solve for the contemporaneous controls in states in which their no-borrowing constraint binds.
} 
FiPIt does not need either nonlinear solvers or interpolation methods for irregular grids. ${ }^{6}$ Standard bi-linear interpolation over rectangular grids still applies.

\subsection{Description of the FiPIt algorithm}

To implement the FiPIt method, we first re-write the equilibrium conditions in recursive form. The model has two endogenous states, $b$ and $k$, and three exogenous states, using $s$ to denote the triple of exogenous shocks $s \equiv(A, R, p)$, which includes the shocks to $\operatorname{TFP}(A)$, the world interest rate $(R)$ and the price of imported inputs $(p)$. The recursive equilibrium is defined by a set of recursive functions for allocations $\left[b^{\prime}(b, k, s), k^{\prime}(b, k, s), c(b, k, s), L(b, k, s), v(b, k, s)\right]$, prices $[w(b, k, s), q(b, k, s), d(b, k, s)]$ and multipliers $[\lambda(b, k, s), \mu(b, k, s)]$ that satisfy the following recursive representation of the equilibrium conditions:

$$
\begin{gathered}
\left(c(b, k, s)-\frac{L(b, k, s)^{\omega}}{\omega}\right)^{-\sigma}=\lambda(b, k, s)(1+\tau) \\
\alpha A k^{\gamma} L(b, k, s)^{\alpha-1} v(b, k, s)^{\eta}=w(b, k, s)\left(1+\phi(R-1)+\frac{\mu(b, k, s)}{\lambda(b, k, s)} \phi R\right) \\
\eta A k^{\gamma} L(b, k, s)^{\alpha} v(b, k, s)^{\eta-1}=p\left(1+\phi(R-1)+\frac{\mu(b, k, s)}{\lambda(b, k, s)} \phi R\right) \\
\lambda(b, k, s)=R \beta E\left[\lambda\left(b^{\prime}(b, k, s), k^{\prime}(b, k, s), s^{\prime}\right)\right]+\mu(b, k, s) \\
\lambda(b, k, s)=\frac{1}{q(b, k, s)} \beta E\left[\lambda ( b ^ { \prime } ( b , k , s ) , k ^ { \prime } ( b , k , s ) , s ^ { \prime } ) \left(d\left(b^{\prime}(b, k, s), k^{\prime}(b, k, s), s^{\prime}\right)\right.\right. \\
d(b, k, s)=\gamma A k^{\gamma-1} L(b, k, s)^{\alpha} v(b, k, s)^{\eta}-\delta+\frac{a}{2} \frac{\left(k^{\prime}(b, k, s)-k\right)^{2}}{k^{2}} \\
q(b, k, s)=1+a\left(\frac{k^{\prime}(b, k, s)-k}{k}\right) \\
w(b, k, s)=L(b, k, s)^{\omega-1}(1+\tau) \\
c(b, k, s)(1+\tau)+k^{\prime}(b, k, s)-(1-\delta) k+\frac{a}{2} \frac{\left(k^{\prime}(b, k, s)-k\right)^{2}}{k}=A k^{\gamma} L(b, k, s)^{\alpha} v(b, k, s)^{\eta} \\
\phi v(R, k, s)-1)\left(L(b, k, s)^{\omega}(1+\tau)+p v(b, k, s)\right)-R^{-1} b^{\prime}(b, k, s)+b
\end{gathered}
$$

\footnotetext{
${ }^{6} \mathrm{~A}$ nonlinear equation may need to be solved for in states in which the credit constraint binds, depending on the structure of the constraint (as we explain in subsection 3.2), but this is separate from the need to solve a two-Euler-equation nonlinear system when time iteration is used to solve models with two endogenous states.
} 
The recursive equilibrium is solved for over a discrete state space, which requires defining discrete grids for $(b, k, s)$. The grid for the shock triples $s \in \mathbf{S}$ comes from the discretization of the stochastic processes of the model's three shocks. This is typically done using Tauchen's quadrature method. Here we take $\mathbf{S}$ and the associated Markov transition probability matrix from Mendoza [2010], where $\mathbf{S}$ has eight triples (i.e. each shock has two realizations). For the endogenous states, we define grids with $M$ nodes for bonds and $N$ nodes for capital, respectively: $\mathbf{B}=\left\{b^{1}<b^{2}<\ldots<b^{M}\right\}$, $\mathbf{K}=\left\{k^{1}<k^{2}<\ldots<k^{N}\right\}$. The state space has $M \times N \times 8$ elements and is defined by all $(b, k, s) \in \mathbf{B} \otimes \mathbf{K} \otimes \mathbf{S}$. Once parameter values and the discrete state space are defined, the FiPIt algorithm is implemented following the setps decribed below.

Step 1. Start iteration $j$ with conjectured functions for the price of capital $\hat{q}_{j}(b, k, s)$, the decision rule for bonds $\hat{B}_{j}(b, k, s) \equiv \hat{b}_{j}^{\prime}(b, k, s)$, and the multiplier ratio $\hat{\tilde{\mu}}_{j}(b, k, s) \equiv \mu_{j}(b, k, s) / \lambda_{j}(b, k, s)$. The first iteration can start with $\hat{\tilde{\mu}}_{0}(b, k, s)=0$ so that the first pass runs as if it were an RBC model and only cases where the constraint binds pass positive multipliers to the next iteration. The initial functions can be set to $\hat{q}_{0}(b, k, s)=1$ and $\hat{B}_{0}(b, k, s)=b$, which imply stationary decision rules for capital and bonds. Note also that this same algorithm can be used to solve a standard RBC model without the occasionally binding constraint, by simply setting $\kappa$ high enough so that the constraint never binds.

Step 2. Compute the iteration- $j$ implied decision rules for capital $\left(K_{j}(b, k, s) \equiv k_{j}^{\prime}(b, k, s)\right)$, consumption, investment (inclusive of adjustment costs), labor, inputs and output as shown below. Note that, given $\hat{q}_{j}(b, k, s)$, the capital decision rule has an analytic solution that follows from optimality condition (28) (i.e. the capital decision rule has a closed-form solution as a function of the price of capital). The factor allocation rules follow from the conditions equating marginal products with marginal costs, which include factor prices and financing costs. The wages bill $w L$ is replaced with $(1+\tau) L^{\omega}$ because of the optimality condition for labor supply. With these arguments in mind, 
the iteration- $j$ implied decision rules are:

$$
\begin{gathered}
K_{j}(b, k, s)=\frac{k}{a}\left[\hat{q}_{j}(b, k, s)-1+a\right] \\
\tilde{i}_{j}(b, k, s)=\left(K_{j}(b, k, s)-k\right)\left[1+\frac{a}{2}\left(\frac{K_{j}(b, k, s)-k}{k}\right)\right]-\delta k \\
v_{j}(b, k, s)=\left\{\frac{A k^{\gamma} \eta^{\frac{\omega-\alpha}{\omega}} \frac{\alpha}{1+\tau} \frac{\alpha}{\omega}}{p^{\frac{\omega-\alpha}{\omega}}\left[1+\phi(R-1)+\hat{\tilde{\mu}}_{j}(b, k, s) \phi R\right]}\right\}^{\frac{\omega}{\omega(1-\eta)-\alpha}} \\
L_{j}(b, k, s)=\left\{\frac{\alpha}{\eta(1+\tau)} p v_{j}((b, k, s)\}^{\frac{1}{\omega}}\right. \\
y_{j}(b, k, s)=A k^{\gamma} L_{j}(b, k, s)^{\alpha} v_{j}(b, k, s)^{\eta}
\end{gathered}
$$

Consumption then follows from the resource constraint:

$$
\begin{aligned}
(1+\tau) c_{j}(b, k, s)= & y_{j}(b, k, s)-p v_{j}(b, k, s)-\phi(R-1)\left[(1+\tau) L_{j}(b, k, s)^{\omega}+p v_{j}(b, k, s)\right] \\
& -\tilde{i}_{j}(b, k, s)-\frac{\hat{B}_{j}(b, k, s)}{R}+b
\end{aligned}
$$

Note that for points where $\hat{\tilde{\mu}}_{j}(b, k, s)=0$, factor allocations and output are the same as for an RBC model without credit frictions, which because of the GHH structure of period utility (i.e. the marginal rate of substitution between $c$ and $L$ is independent of $c$ ) depend only on $(k, s)$. We keep them as functions of all three states because when $\hat{\tilde{\mu}}_{j}(b, k, s)>0$ factor allocations and output do depend on the three states.

Step 3. Assume the collateral constraint does not bind. This implies that the new decision rule for the modified multiplier is $\hat{\tilde{\mu}}_{j+1}(b, k, s)=0$, and the decision rules for the rest of the endogenous variables are solved as follows:

3.1 Factor allocations and output again match the expressions corresponding to an RBC model with perfect credit markets:

$$
\begin{gathered}
v_{j+1}(b, k, s)=\left\{\frac{A k^{\gamma} \eta^{\frac{\omega-\alpha}{\omega}} \frac{\alpha}{1+\tau} \frac{\alpha}{\omega}}{p^{\frac{\omega-\alpha}{\omega}}[1+\phi(R-1)]}\right\}^{\frac{\omega}{\omega(1-\eta)-\alpha}} \\
L_{j+1}(b, k, s)=\left\{\frac{\alpha}{\eta(1+\tau)} p v_{j+1}(b, k, s)\right\}^{\frac{1}{\omega}} \\
y_{j+1}(b, k, s)=A k^{\gamma} L_{j+1}(b, k, s)^{\alpha} v_{j+1}(b, k, s)^{\eta}
\end{gathered}
$$

3.2 Solve for $c_{j+1}$ by applying the fixed-point iteration method to the Euler equation for bonds. 
The iteration- $j$ conjectures for capital and bonds are used everywhere in the right-hand-side of this Euler equation, so that we obtain an analytic solution for $c_{j+1}$. Keep track of the subscripts denoting which function is used in each term:

$$
\begin{aligned}
& c_{j+1}(b, k, s) \\
& =\left\{\beta R E\left[\left(c_{j}\left(\hat{B}_{j}(b, k, s), K_{j}(b, k, s), s^{\prime}\right)-\frac{L_{j}\left(\hat{B}_{j}(b, k, s), K_{j}(b, k, s), s^{\prime}\right)^{\omega}}{\omega}\right)^{-\sigma}\right]\right\}^{-\frac{1}{\sigma}} \\
& \quad+\frac{L_{j+1}(b, k, s)^{\omega}}{\omega}
\end{aligned}
$$

In the above expression, the functions $c_{j}(b, k, s)$ and $L_{j}(b, k, s)$ are defined only at the nodes of $\mathbf{B} \otimes \mathbf{K} \otimes \mathbf{S}$, but since the values of $\hat{B}_{j}(b, k, s)$ and $K_{j}(b, k, s)$ generally do not match node grids in $\mathbf{B}$ and $\mathbf{K}$, respectively, $c_{j}(\cdot)$ and $L_{j}(\cdot)$ are interpolated over their first two arguments to determine $c_{j}\left(\hat{B}_{j}(b, k, s), K_{j}(b, k, s), s^{\prime}\right)$ and $L_{j}\left(\hat{B}_{j}(b, k, s), K_{j}(b, k, s), s^{\prime}\right)$. Standard bi-linear interpolation is applied. Use extrapolation if $K_{j}(b, k, s)$ is below (above) $k^{1}\left(k^{N}\right)$ and also if $\hat{B}_{j}(b, k, s)$ is above $b^{M}$, but for $\hat{B}_{j}(b, k, s)<b^{1}$ evaluate the functions at $b^{1}$, because the lower bound on bonds represents an ad-hoc debt limit commonly used for calibration of the model to the data (see Durdu et al. [2019]). Note also that, because of the fractional exponent (since typically $\sigma>1$ ) the above equation solves only if $c_{j}(\cdot)-\frac{L_{j}(\cdot)^{\omega}}{\omega}>0$, but if this is true for the consumption and labor decision rules implied by the initial conjectures set for the first iteration $\left(c_{0}(\cdot), L_{0}(\cdot)\right)$, it will also be true at any iteration $j>0$.

3.3 Solve for $B_{j+1}(b, k, s)$ using the resource constraint:

$$
\begin{array}{r}
B_{j+1}(b, k, s)=R\left\{y_{j+1}(b, k, s)-p v_{j+1}(b, k, s)\right. \\
-\phi(R-1)\left[(1+\tau) L_{j+1}(b, k, s)^{\omega}+p v_{j+1}(b, k, s)\right]-\tilde{i}_{j}(b, k, s) \\
\left.-(1+\tau) c_{j+1}(b, k, s)+b\right\}
\end{array}
$$

3.4 Evaluate if the collateral constraint binds. If:

$$
\frac{B_{j+1}(b, k, s)}{R}-\phi R\left[(1+\tau) L_{j+1}(b, k, s)^{\omega}+p v_{j+1}(b, k, s)\right]+\kappa \hat{q}_{j}(b, k, s) K_{j}(b, k, s) \geq 0,
$$

the constraint does not bind at the point $(b, k, s)$, the functions with $j+1$ subscripts are saved, and skip to Step 5. Otherwise, the constraint binds at this point, the functions with $j+1$ subscripts are discarded and move to Step 4. 
Step 4. Solve for decision rules when the collateral constraint binds. Since $\hat{q}_{j}(b, k, s)$ has not changed, we use the same iteration- $j$ implied decision rule for capital $K_{j}(b, k, s)=\frac{k}{a}\left[\hat{q}_{j}(b, k, s)-1+a\right]$ and the same function $\tilde{i}_{j}(b, k, s)$ as before. This is the most computationally intensive step, because it solves a nonlinear simultaneous equations system to determine $L_{j+1}(b, k, s), v_{j+1}(b, k, s), c_{j+1}(b, k, s)$, $B_{j+1}(b, k, s), \tilde{\mu}_{j+1}(b, k, s)$. The five equations in the system are the two optimality conditions for factor allocations, the Euler equation for bonds (with the $\tilde{\mu}$ terms), the credit constraint holding with equality, and the resource constraint. To make the solution more tractable, we express $L_{j+1}(b, k, s), v_{j+1}(b, k, s), c_{j+1}(b, k, s), B_{j+1}(b, k, s)$ as functions of $\tilde{\mu}(b, k, s)$, and use the results to reduce the system to a single nonlinear equation in $\tilde{\mu}(b, k, s)$. In the simplified system, factor allocations, consumption and bonds are functions denoted $L_{j+1}(b, k, s, \tilde{\mu}), v_{j+1}(b, k, s, \tilde{\mu}), c_{j+1}(b, k, s, \tilde{\mu})$, $B_{j+1}(b, k, s, \tilde{\mu})$, but to make the notation simpler we write them as depending on $\tilde{\mu}$ only (still, keep in mind the set of equations needs to be solved for each $(b, k, s)$ for which the constraint was found to be binding in step $\mathbf{3 . 4}$ ):

$$
\begin{gathered}
v(\tilde{\mu})=\left\{\frac{A k^{\gamma} \eta^{\frac{\omega-\alpha}{\omega}} \frac{\alpha}{1+\tau} \frac{\alpha}{\omega}}{p^{\frac{\omega-\alpha}{\omega}}[1+\phi(R-1)+\tilde{\mu} \phi R]}\right\}^{\frac{\omega}{\omega(1-\eta)-\alpha}} \\
L(\tilde{\mu})=\left\{\frac{\alpha}{\eta(1+\tau)} p v(\tilde{\mu})\right\}^{\frac{1}{\omega}} \\
\frac{B(\tilde{\mu})}{R}=-\kappa \hat{q}_{j} K_{j}+\phi R p v(\tilde{\mu})\left[1+\frac{\alpha}{\eta}\right] \\
(1+\tau) c(\tilde{\mu})=y(\tilde{\mu})-p v(\tilde{\mu})-\phi(R-1) p v(\tilde{\mu})\left[1+\frac{\alpha}{\eta}\right]-\tilde{i}_{j}-\frac{B(\tilde{\mu})}{R}+b
\end{gathered}
$$

where $y(\tilde{\mu})=A k^{\gamma} L(\tilde{\mu})^{\alpha} v(\tilde{\mu})^{\eta}$. The equations for labor and inputs follow from combining the borrowing constraint with the optimality conditions equating marginal products with marginal costs, including the $\tilde{\mu}$ terms. They are the same equations used in Step 2, but now we need to find the value of $\tilde{\mu}_{j+1}$ that solves them, instead of taking as given $\tilde{\mu}_{j}$.

In addition to equations (43)-(46), the solution for $\tilde{\mu}_{j+1}(b, k, s)$ must also satisfy the Euler equation for bonds, which can be written as:

$$
\tilde{\mu}_{j+1}(b, k, s)=1-\frac{\beta R E\left[\left(c_{j}\left(\hat{B}_{j}(b, k, s), K_{j}(b, k, s), s^{\prime}\right)-\frac{L_{j}\left(\hat{B}_{j}(b, k, s), K_{j}(b, k, s), s^{\prime}\right)^{\omega}}{\omega}\right)^{-\sigma}\right]}{\left(c\left(\tilde{\mu}_{j+1}(b, k, s)\right)-\frac{L\left(\tilde{\mu}_{j+1}(b, k, s)\right)^{\omega}}{\omega}\right)^{-\sigma}}
$$

Notice the numerator in the second term in the right-hand-side still applies fixed-point iteration by 
computing expected marginal utility using j-dated functions only. The values of $c_{j}\left(\hat{B}_{j}(b, k, s), K_{j}(b, k, s), s^{\prime}\right)$ and $L_{j}\left(\hat{B}_{j}(b, k, s), K_{j}(b, k, s), s^{\prime}\right)$ are again determined by bi-linear interpolation.

Algebraic manipulation of equations (43)-(47) reduces to this nonlinear equation in $\tilde{\mu}_{j+1}(\cdot)$

$$
\begin{aligned}
& \left(1-\tilde{\mu}_{j+1}(\cdot)\right)\left\{C_{1}^{\frac{\omega}{(1-\eta) \omega-\alpha}}\left[\frac{\alpha}{1+\phi(R-1)+\tilde{\mu}_{j+1}(\cdot) \phi R}\right]^{\frac{\eta \omega+\alpha}{(1-\eta) \omega-\alpha}}\right. \\
& \left.-\left[\frac{\alpha C_{1}}{1+\phi(R-1)+\tilde{\mu}_{j+1}(\cdot) \phi R}\right]^{\frac{\omega}{(1-\eta) \omega-\alpha}} C_{2}-\left(\frac{\tilde{i}_{j}(\cdot)-\kappa \hat{q}_{j}(\cdot) K_{j}(\cdot)-b}{1+\tau}\right)\right\}^{-\sigma} \\
& =\beta R E\left[\left(c_{j}\left(\hat{B}_{j}(\cdot), K_{j}(\cdot), s^{\prime}\right)-\frac{L_{j}\left(\hat{B}_{j}(\cdot), K_{j}(\cdot), s^{\prime}\right)^{\omega}}{\omega}\right)^{-\sigma}\right]
\end{aligned}
$$

where:

$$
\begin{gathered}
C_{1} \equiv\left(\frac{1}{1+\tau}\right)^{1-\eta} A k^{\gamma}\left(\frac{\eta}{\alpha p}\right)^{\eta} \\
C_{2} \equiv \frac{1}{\omega}+\frac{\eta}{\alpha}+\phi\left(1+\frac{\eta}{\alpha}\right)(2 R-1)
\end{gathered}
$$

Note again that, because of the fractional exponent in the right-hand-side of (48), the equation solves only if $c_{j}(\cdot)-\frac{L_{j}(\cdot)^{\omega}}{\omega}>0$. Since the first iteration starts with $\tilde{\mu}_{0}(\cdot)=0$, any state that yields a binding credit constraint in the first iteration will solve for $\tilde{\mu}_{1}(\cdot)$ as long as the same condition required for the unconstrained consumption function (eq. (40)) to solve in the first iteration holds, namely that $c_{0}(\cdot)-\frac{L_{0}(\cdot)^{\omega}}{\omega}>0$ for the decision rules implied by the initial conjectures set for the first iteration. Moreover, since when the constraint binds it must be true that $0<\tilde{\mu}<1$, it follows from eq. (47) that $c_{j}(\cdot)-\frac{L_{j}(\cdot)^{\omega}}{\omega}>0$ will hold for any iteration $j>0$. Once $\tilde{\mu}_{j+1}(b, k, s)$ is solved, the functions $v_{j+1}(b, k, s), L_{j+1}(b, k, s), B_{j+1}(b, k, s), c_{j+1}(b, k, s)$ are determined using equations (43)(46), but replacing $\tilde{\mu}$ with $\tilde{\mu}_{j+1}(b, k, s)$. The functions with $j+1$ subscripts are saved, and we move to Step 5.

It is important to note that, depending on the structure of the occasionally binding constraint, if $\tilde{\mu}$ can be solved for recursively after solving for the allocations, Step 4 is much easier because FiPIt does not require a non-linear solver anywhere. For example, if working capital is not in the credit constraint, we can set $B_{j+1}(b, k, s) / R=-\kappa \hat{q}_{j}(b, k, s) K_{j}(b, k, s)$, and this can be used to determine $c_{j+1}(b, k, s)$ directly from the resource constraint. The implied value of $\tilde{\mu}_{j+1}(b, k, s)$ can then be solved for from the bonds Euler equation. The same applies for a credit constraint set to a constant value, as in Ludwig and Schön [2018], where they used $b_{t+1} \geq 0$. Hence, FiPIt can solve models with a large class of occasionally binding constraints without using a non-linear solver at any point, 
whereas the Ludwig-Schön algorithm needs both the Delaunay interpolation and a non-linear solver when the constraint binds.

Step 5. Return to Step 3 and repeat $\forall(b, k, s) \in \mathbf{B} \otimes \mathbf{K} \otimes \mathbf{S}$. This is necessary before proceeding to compute a new asset pricing function, because the complete set of $\mathrm{j}+1$-dated functions is required.

Step 6. Compute the new pricing function $q_{j+1}(b, k, s)$. We describe two ways of doing this:

6.1 The FiPIt algorithm proceeds in a manner analogous to fixed-point iteration on the Euler equation for bonds, by applying the new decision rules for $c_{j+1}(\cdot), L_{j+1}(\cdot), B_{j+1}(\cdot), \tilde{\mu}_{j+1}(\cdot)$ to the Euler equation for capital and solving it so as to obtain the following analytic solution for $q_{j+1}(b, k, s)$ :

$$
\begin{aligned}
& q_{j+1}(b, k, s) \\
& =\frac{\beta E_{t}\left[\left(c_{j+1}\left(B_{j+1}(\cdot), K_{j}(\cdot), s^{\prime}\right)-\frac{L_{j+1}\left(B_{j+1}(\cdot), K_{j}(\cdot), s^{\prime}\right)^{\omega}}{\omega}\right)^{-\sigma}\left[d^{\prime}(\cdot)+\hat{q}_{j}\left(B_{j+1}(\cdot), K_{j}(\cdot), s^{\prime}\right)\right]\right]}{\left(c_{j+1}(\cdot)-\frac{L_{j+1}(\cdot)^{\omega}}{\omega}\right)^{-\sigma}\left(1-\kappa \tilde{\mu}_{j+1}(\cdot)\right)}
\end{aligned}
$$

The asset price used in the right-hand-side is the conjecture set in Step 1. Since all the functions in the right-hand-side are known, the equation solves directly for $q_{j+1}(b, k, s)$. The values of $\left.c_{j+1}\left(B_{j+1}(\cdot), K_{j}(\cdot), s^{\prime}\right)\right), L_{j+1}\left(B_{j+1}(\cdot), K_{j}(\cdot), s^{\prime}\right)$ and $\hat{q}_{j}\left(B_{j+1}(\cdot), K_{j}(\cdot), s^{\prime}\right)$ are determined by bi-linear interpolation. The dividends function is obtained from equation (27) using bi-linear interpolation on its components (see Appendix for details). Notice that the decision rule for bonds that sets the value of $b_{t+1}$ at which these functions are interpolated is a $\mathrm{j}+1$-dated function, not the $\mathrm{j}$-dated function used in Steps $\mathbf{3}$ and $\mathbf{4}$, but over the capital dimension we are still using the $\mathrm{j}$-dated decision rule.

6.2 A variant of the algorithm labeled Fixed-Point Iteration with Forward Solution (FPIFS) solves for the new price conjecture by iterating to convergence on the capital Euler equation (i.e. it uses the forward solution of the asset price). Index the iterations on this equation with superscript $z$, the iterations solve this functional equation problem, always using the $j+1$ - 
dated functions and the multiplier $\tilde{\mu}_{j+1}(\cdot)$ obtained in Steps $\mathbf{3}$ to $\mathbf{5}$ :

$$
\begin{aligned}
& q^{z+1}(b, k, s) \\
& =\frac{\beta E_{t}\left[\left(c_{j+1}\left(B_{j+1}(\cdot), K_{j}(\cdot), s^{\prime}\right)-\frac{L_{j+1}\left(B_{j+1}(\cdot), K_{j}(\cdot), s^{\prime}\right)^{\omega}}{\omega}\right)^{-\sigma}\left[d^{\prime}(\cdot)+q^{z}\left(B_{j+1}(\cdot), K_{j}(\cdot), s^{\prime}\right)\right]\right]}{\left(c_{j+1}(\cdot)-\frac{L_{j+1}(\cdot) \omega}{\omega}\right)^{-\sigma}\left(1-\kappa \tilde{\mu}_{j+1}(\cdot)\right)}
\end{aligned}
$$

Iterate until $\left\|q^{z+1}(b, k, s)-q^{z}(b, k, s)\right\| \leq \varepsilon^{q}$ for small $\varepsilon^{q}$, and if the result converges, the final result sets $q_{j+1}(b, k, s)$. The values of $\left.c_{j+1}\left(B_{j+1}(\cdot), K_{j}(\cdot), s^{\prime}\right)\right), L_{j+1}\left(B_{j+1}(\cdot), K_{j}(\cdot), s^{\prime}\right), d^{\prime}(\cdot)$ and $q^{z}\left(B_{j+1}(\cdot), K_{j}(\cdot), s^{\prime}\right)$ are determined using bi-linear interpolation as in step 6.1.

Step 7. Check the convergence of the conjectured functions. Convergence requires that for small $\varepsilon^{f}$ the following conditions are satisfied $\forall(b, k, s) \in \mathbf{B} \otimes \mathbf{K} \otimes \mathbf{S}$ :

$$
\begin{gathered}
\left|q_{j+1}(b, k, s)-\hat{q}_{j}(b, k, s)\right| \leq \varepsilon^{f} \\
\left|B_{j+1}(b, k, s)-\hat{B}_{j}(b, k, s)\right| \leq \varepsilon^{f} \\
\left|\tilde{\mu}_{j+1}(b, k, s)-\hat{\tilde{\mu}}_{j}(b, k, s)\right| \leq \varepsilon^{f}
\end{gathered}
$$

If these conditions hold, the recursive competitive equilibrium has been solved. The level of the multiplier on the credit constraint can then be solved for as follows:

$$
\mu_{j+1}(b, k, s)=\tilde{\mu}_{j+1}(b, k, s)\left(c_{j+1}(b, k, s)-\frac{L_{j+1}(b, k, s)^{\omega}}{\omega}\right)^{-\sigma}
$$

The accuracy of the solution can then be evaluated by verifying that the equilibrium conditions hold, including computations of the maximum and average absolute values of the errors in the Euler equations of $k$ and $b$.

If any of the three convergence conditions fails, update the conjectured functions using a convex combination of the last conjectures and the new functions to dampen possible overshooting or speed up convergence. This is conventional practice because there is no guarantee that fixed-point iteration algorithms converge, but when they diverge it is generally because they overshoot the true solution. Hence, the new conjectures are set as:

$$
\hat{x}_{j+1}(b, k, s)=\left(1-\rho^{x}\right) \hat{x}_{j}(b, k, s)+\rho^{x} x_{j+1}(b, k, s)
$$

for $x=[q, B, \tilde{\mu}]$ and some $0 \leq \rho^{x}$. Notice that $\hat{x}_{j}(b, k, s)$ in the right-hand-side of this expression represents the initial conjectures that were used in the current iteration, while $\hat{x}_{+1} j(b, k, s)$ in the 
left-hand-side denotes the new conjectures for the next iteration. Use $0<\rho^{x}<1\left(\rho^{x}>1\right)$ for the particular function $x(\cdot)$ that is not converging (converging too slowly). Return to Step 2, setting $\hat{x}_{j}(b, k, s)=\hat{x}_{j+1}(b, k, s)$, and repeat until convergence is attained.

\section{RBC \& Sudden Stops Examples}

We developed a set of Matlab programs to implement the FiPIt algorithm and used them to solve the RBC and Sudden Stops models. The models' parameter values are taken from Mendoza [2010] and listed in Table 1. We also use the same Markov process for the models' three shocks. The only difference, as mentioned earlier, is that instead of using preferences with an endogenous rate of time preference we use standard time-separable expected utility with constant discounting, setting the subjective discount factor at $\beta=0.92$.

We report the results of solutions using FiPIt and the FPIFS variant, as well as solutions from three other algorithms: TIFS replaces the fixed-point iteration solution of the bonds decision rule with a standard time iteration solution that uses a non-linear solver, and solves for the price of capital using the forward solution of the capital Euler equation; TIFPI uses again standard time iteration for the bonds decision rule, but solves for the price of capital using the fixed-point iteration approach; and FTI is the full time iteration solution in which the Euler equations for capital and bonds are solved as a non-linear equation system. All the computations were made using Matlab R2017a on a Windows 10 laptop with an Intel Core i7-6700HQ 2.60GHz four-core chip and 16GB of RAM. The Matlab codes and an Appendix that explains how the codes implement each of the FiPIt algorithm steps are available online.

The state space of the RBC (SS) solutions consists of evenly-spaced grids with 80 (72) nodes for bonds and 30 nodes for capital. $\mathbf{K}$ spans the [654.5,885.5] interval while in the RBC (SS) solutions B spans the [-300.0,800.0] ([-188.6,800.0]) interval. Solving with larger grids increases sharply execution time and produces negligibly different results, while solving with smaller grids is faster but yields inaccurate results. The Markov process of the shocks has two realizations for each shock and their values together with the associated $8 \times 8$ transition probability matrix approximate the variability, autocorrelation, and contemporaneous correlation of TFP, interest rates and the price of imported inputs in the data (see Mendoza [2010] for details). In all the solutions except FTI, we found faster convergence by setting the dampening parameters for updating the conjectured functions to 0.3 for the price of capital ( 0.25 for a scenario with 60 capital nodes) and 1 for bonds 
and $\tilde{\mu}$. For FTI solutions, we kept $\rho^{x}=1$ for all three functions, and confirmed that these produces convergence in the smallest number of iterations.

\begin{tabular}{|c|c|}
\hline Parameter & Value \\
\hline$\sigma \quad$ risk aversion & 2.0 \\
\hline$\omega$ labor elasticity & 1.8461 \\
\hline discount factor & 0.92 \\
\hline capital adjustment cost & 2.75 \\
\hline working capital parameter & 0.2579 \\
\hline depreciation rate & 0.088 \\
\hline labor share & 0.59 \\
\hline imported inputs share & 0.10 \\
\hline$\gamma$ capital share of income & 0.31 \\
\hline$\tau$ tax on consumption & 0.17 \\
\hline$A$ Average TFP & 6.982 \\
\hline
\end{tabular}

\subsection{Results for the RBC Model}

Table 2 compares long-run moments of the main macro aggregates and performance statistics for the RBC model across seven solution scenarios. The RBC case is solved by setting $\kappa$ high enough so that the credit constraint never binds. In this case, $\kappa=1$ was sufficient. The Table includes results for all the algorithm variations mentioned earlier. Columns (1) and (2) are FiPIt solutions with $80 \times 60$ v. $80 \times 30$ grids respectively, (3) is the TFIS solution, (4) is the FPIFS solution, (5) is the TIFPI solution, and (6) is the FTI solution. Column (7) shows the results reported in Mendoza [2010].

The moments reported in Columns (1) to (6) are very similar, and in fact identical up to one or two decimals. The comparison of Cols. (1) and (2) shows that solving using FiPIt with the smaller capital grid has nearly no effect on the results but reduces execution time sharply. Comparisons of Cols. (2)-(5) v. Col. (6) show that all those solution alternatives yield effectively the same results as the FTI method. The results from Mendoza [2010] in Column (7) are qualitatively similar in terms of ranking of volatilities and signs and ranking of correlations and autocorrelations, but quantitatively shows more differences. These are due to the different discount factors (Mendoza used endogenous discounting) and the different solution methods (Mendoza solved forcing decision rules to be on the nodes of the grids of bonds and capital, instead of using interpolation, and used value function iteration on a quasi planner's problem with $w$ and $q$ restricted to satisfy the labor supply and investment optimality conditions). 
The performance metrics for Columns (1)-(6) reported in panel (b) of Table 2 show that all the solutions have similar accuracy, with very small maximum and average absolute-value errors in the Euler equations for bonds and capital. In terms of execution time, however, the FiPIt method in Col. (2) dominates the other solution methods by large margins. The absolute speeds will vary widely with hardware and software configurations, but the relative speeds are likely to vary less and the ranking across methods based on this criterion is unlikely to change. Comparing speeds relative to FiPIt, the second fastest method is FPIFS in Col. (4), which takes twice as long to solve. This algorithm only differs from FiPIt in that it solves for the price of capital by solving forward the capital Euler equation. The slowest methods are the three that use time iteration (i.e. a nonlinear solver) at least partially. In Cols. (3) and (5) the bonds decision rule is solved with the time iteration method, but the price of capital is solved using the forward solution in Col. (3) v. fixed-point iteration in Col. (5). This makes little difference in execution time, as they take roughly 34 and 36 times as long as the FiPIt solution in Col. (2), respectively. Interestingly, the standard FTI method in Col. (6), which solves the simultaneous nonlinear Euler equations for bonds and capital, is significantly faster than the methods used in Cols. (3) and (5), indicating that solving only one nonlinear Euler equation instead of two does not guarantee a faster algorithm. Still, the FTI execution time exceeds that of the FiPIt solution by a factor of 18.1 !

The FTI solution is faster than the ones in Cols. (3) and (5) because time iteration takes advantage of the contraction mapping properties of the two nonlinear Euler equations by solving them simultaneously while fixed-point iteration methods do not. Intuitively, every iteration with FTI tends to generate relatively more accurate outcomes, and hence attains convergence in 94 iterations. The algorithms in Cols. (3) and (5) take more than twice as many iterations (196 iterations for TIFS and 213 for TIFPI), and still in each they have to use a root finder because they solve for the bonds decision rule using time iteration. The FiPIt method converges in a similar number of iterations (201) as these two methods, but goes through each iteration much faster because it avoids using nonlinear solvers, overcoming the drawback of not taking advantage of the contraction mapping properties of the Euler equations, and this makes it the fastest method. FPIFS in Col. (4) is the second fastest for a similar reason, and it is slower than FiPIt because solving the price of capital with the forward solution is slower than with fixed-point iteration. 
Table 2: Long-run Moments \& Performance Metrics: RBC Model Solutions

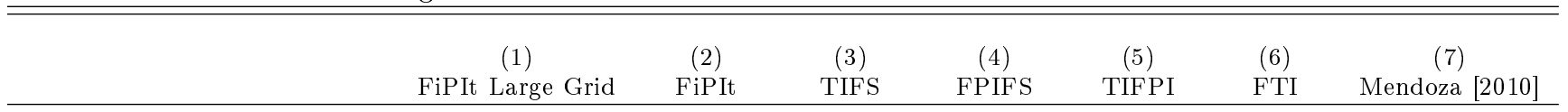

(a) Long-run moments

\begin{tabular}{|c|c|c|c|c|c|c|c|}
\hline \multicolumn{8}{|l|}{ Mean } \\
\hline$g d p$ & 393.803 & 393.847 & 393.850 & 393.847 & 393.850 & 393.813 & 390.135 \\
\hline$c$ & 263.846 & 264.021 & 263.901 & 264.023 & 263.899 & 263.871 & 263.152 \\
\hline$i$ & 67.523 & 67.530 & 67.530 & 67.529 & 67.530 & 67.518 & 66.203 \\
\hline$n x / g d p$ & 0.046 & 0.045 & 0.046 & 0.045 & 0.046 & 0.046 & 0.042 \\
\hline$k$ & 765.720 & 765.885 & 765.895 & 765.884 & 765.896 & 765.759 & 752.27 \\
\hline$b / g d p$ & -0.378 & -0.372 & -0.377 & -0.372 & -0.377 & -0.377 & -0.326 \\
\hline$q$ & 1.000 & 1.000 & 1.000 & 1.000 & 1.000 & 1.000 & 1.000 \\
\hline leverage ratio & -0.288 & -0.286 & -0.288 & -0.286 & -0.288 & -0.288 & -0.266 \\
\hline$v$ & 42.645 & 42.649 & 42.650 & 42.649 & 42.650 & 42.646 & 42.247 \\
\hline working capital & 76.708 & 76.716 & 76.717 & 76.716 & 76.717 & 76.710 & 75.993 \\
\hline \multicolumn{8}{|l|}{ Standard deviation (in percent) } \\
\hline$g d p$ & 3.97 & 3.99 & 3.99 & 3.99 & 3.99 & 3.99 & 3.90 \\
\hline$c$ & 5.12 & 5.15 & 5.14 & 5.15 & 5.14 & 5.14 & 4.21 \\
\hline$i$ & 13.50 & 13.51 & 13.50 & 13.51 & 13.50 & 13.51 & 13.85 \\
\hline$n x / g d p$ & 3.51 & 3.53 & 3.52 & 3.53 & 3.52 & 3.52 & 3.00 \\
\hline$k$ & 4.60 & 4.65 & 4.65 & 4.65 & 4.65 & 4.65 & 4.39 \\
\hline$b / g d p$ & 29.94 & 30.28 & 30.07 & 30.28 & 30.06 & 30.04 & 17.57 \\
\hline$q$ & 3.23 & 3.24 & 3.23 & 3.24 & 3.23 & 3.23 & 3.33 \\
\hline leverage ratio & 14.26 & 14.42 & 14.32 & 14.42 & 14.32 & 14.31 & 8.32 \\
\hline$v$ & 5.90 & 5.91 & 5.91 & 5.91 & 5.91 & 5.91 & 5.85 \\
\hline working capital & 4.38 & 4.40 & 4.40 & 4.40 & 4.40 & 4.40 & 4.32 \\
\hline \multicolumn{8}{|l|}{ Correlation with GDP } \\
\hline$g d p$ & 1.000 & 1.000 & 1.000 & 1.000 & 1.000 & 1.000 & 1.000 \\
\hline$c$ & 0.775 & 0.773 & 0.776 & 0.773 & 0.776 & 0.776 & 0.861 \\
\hline$i$ & 0.645 & 0.640 & 0.640 & 0.640 & 0.640 & 0.640 & 0.616 \\
\hline$n x / g d p$ & -0.234 & -0.227 & -0.229 & -0.227 & -0.229 & -0.230 & -0.191 \\
\hline$k$ & 0.765 & 0.767 & 0.767 & 0.767 & 0.767 & 0.767 & 0.756 \\
\hline$b / g d p$ & 0.091 & 0.090 & 0.093 & 0.090 & 0.093 & 0.093 & -0.023 \\
\hline$q$ & 0.391 & 0.381 & 0.381 & 0.381 & 0.381 & 0.381 & 0.379 \\
\hline leverage ratio & 0.113 & 0.112 & 0.115 & 0.112 & 0.115 & 0.115 & 0.001 \\
\hline$v$ & 0.833 & 0.834 & 0.834 & 0.834 & 0.834 & 0.834 & 0.830 \\
\hline working capital & 0.995 & 0.995 & 0.995 & 0.995 & 0.995 & 0.995 & 0.995 \\
\hline \multicolumn{8}{|l|}{ First-order autocorrelation } \\
\hline$g d p$ & 0.829 & 0.830 & 0.830 & 0.830 & 0.830 & 0.830 & 0.822 \\
\hline$c$ & 0.884 & 0.885 & 0.885 & 0.885 & 0.885 & 0.885 & 0.817 \\
\hline$i$ & 0.516 & 0.516 & 0.516 & 0.516 & 0.516 & 0.516 & 0.493 \\
\hline$n x / g d p$ & 0.708 & 0.711 & 0.710 & 0.711 & 0.710 & 0.709 & 0.549 \\
\hline$k$ & 0.967 & 0.964 & 0.964 & 0.964 & 0.964 & 0.964 & 0.962 \\
\hline$b / g d p$ & 0.996 & 0.997 & 0.997 & 0.997 & 0.997 & 0.997 & 0.175 \\
\hline$q$ & 0.458 & 0.460 & 0.460 & 0.460 & 0.460 & 0.460 & 0.440 \\
\hline leverage ratio & 0.997 & 0.997 & 0.997 & 0.997 & 0.997 & 0.997 & 0.083 \\
\hline$v$ & 0.780 & 0.780 & 0.780 & 0.780 & 0.780 & 0.780 & 0.776 \\
\hline working capital & 0.807 & 0.807 & 0.808 & 0.807 & 0.808 & 0.808 & 0.801 \\
\hline \multicolumn{8}{|c|}{ (b) Performance metrics } \\
\hline Max Log10 Abs. Euler Error & -6.93 & -6.93 & -6.93 & -6.93 & -6.93 & -6.93 & - \\
\hline At Grid Points $(b, k, s)$ & $(1,1,3)$ & $(1,1,3)$ & $(1,1,3)$ & $(1,1,3)$ & $(1,1,3)$ & $(1,1,3)$ & - \\
\hline Mean Log10 Abs. Euler Error & -16.20 & -15.96 & -13.37 & -15.80 & -13.36 & -13.33 & - \\
\hline \multicolumn{8}{|l|}{ Capital Euler Equation } \\
\hline Max Log10 Abs. Euler Error & -15.18 & -15.42 & -15.43 & -15.43 & -15.42 & -9.91 & - \\
\hline At Grid Points $(b, k, s)$ & $(23,1,7)$ & $(24,1,7)$ & $(24,1,7)$ & $(24,1,7)$ & $(23,1,7)$ & $(2,1,3)$ & - \\
\hline Mean Log10 Abs. Euler Error & -16.36 & -16.36 & -16.21 & -16.19 & -16.38 & -12.74 & - \\
\hline Grid size $(\# b, \# k)$ & $(80,60)$ & $(80,30)$ & $(80,30)$ & $(80,30)$ & $(80,30)$ & $(80,30)$ & $(80,60)$ \\
\hline Seconds elapsed & 160 & 100 & 3367 & 178 & 3643 & 1808 & - \\
\hline Relative to FiPIt & 1.6 & 1.0 & 33.7 & 1.8 & 36.4 & 18.1 & - \\
\hline Number of iterations & 214 & 201 & 196 & 184 & 213 & 94 & - \\
\hline
\end{tabular}

Note: Column (1) and Column (2) are for the FiPIt algorithm, fixed-point iteration is used for both the bonds decision rule and the price of capital. Column (3) is for the TIFS method, which uses the time iteration method for the bonds decision rule and the forward solution of the capital Euler equation for the price of capital. Column (4) is for the FPIFS method, which uses fixed-point iteration for the bonds decision rule and the forward solution of the capital Euler equation for the price of capital. Column (5) is for the TIFPI method, which uses time iteration for the decision rule for bonds and fixed-point iteration for the price of capital. Column (6) is for the FTI method, which solves the bonds decision rule and the price of capital by solving the Euler equations for bonds and capital as two simultaneous non-linear equations. 
Figure 1 shows the ergodic marginal distributions of $b$ and $k$, and the ergodic joint marginal distribution of $(b, k)$ produced by the FiPIt solution. To compute the full limiting distribution, we applied the standard procedure iterating to convergence on the law of motion of the conditional distribution of $(b, k, s)$ starting from an arbitrary initial condition. Full details are provided in the Appendix. The distributions produced by all the other solution methods, includings FTI, are visually identical, and hence we only show the ones for the FiPIt case. The long-run moments listed in Table 2 were produced using this limiting distribution.

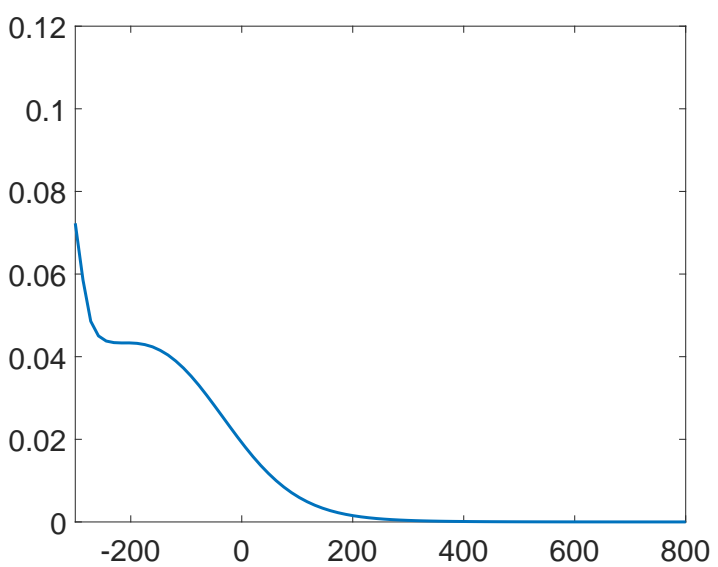

(a) Ergodic Marginal Distribution of Bonds

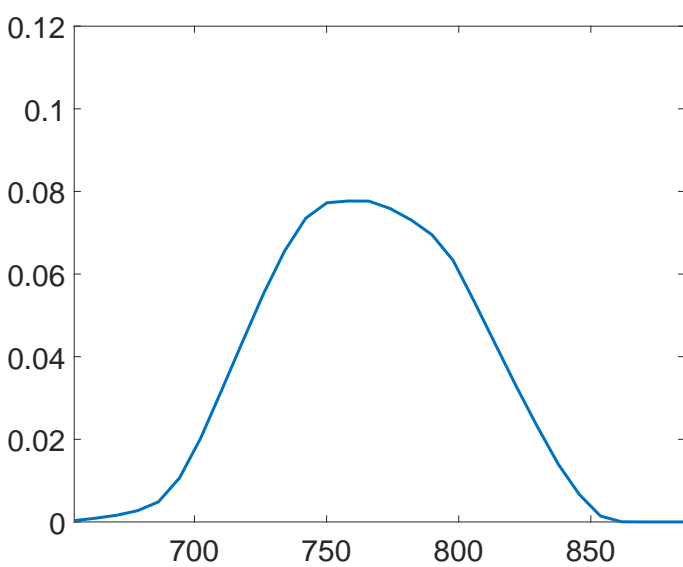

(b) Ergodic Marginal Distribution of Capital

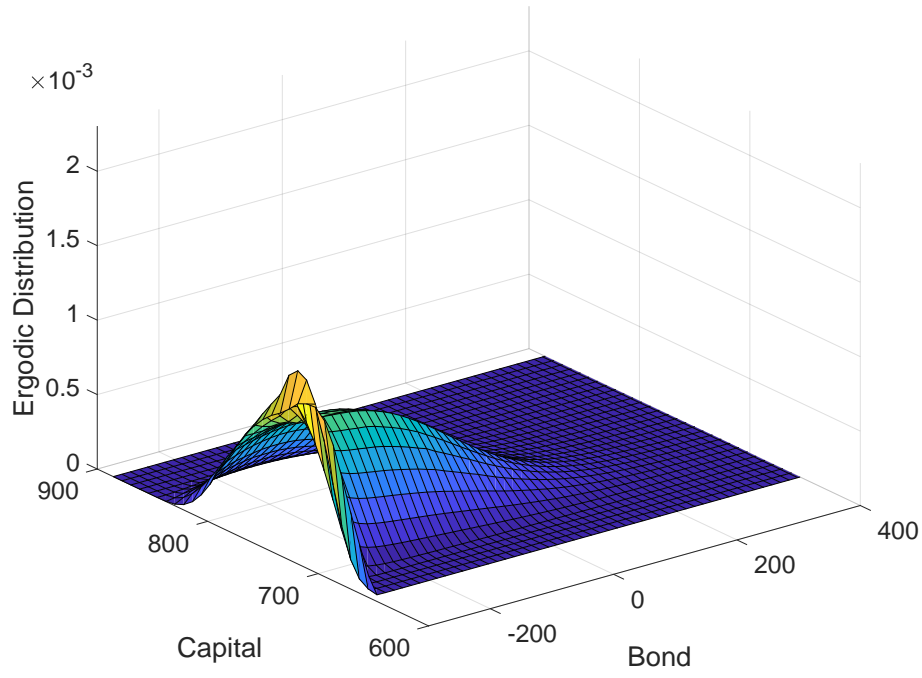

(c) Ergodic Distribution of Bonds and Capital

Figure 1: Long-run Distributions of the RBC model solved with FiPIt. 
The distributions show standard properties of the small open economy RBC model. The distribution for capital is bell-shaped, while the one for bonds is skewed to the left. This occurs because the model inherits the property of incomplete markets models with standard preferences requiring $\beta R<1$ in order to prevent precautionary saving incentives from driving bond holdings to infinity. As a result, the distribution of bond holdings has mass at the pre-determined ad-hoc debt limit defined by the lower bound of the bonds grid, or at the natural debt limit defined by the annuity value of the lowest disposable income realization.

\subsection{Results for the Sudden Stops Model}

Table 3 shows long-run moments and performance metrics for the SS model with $\kappa=0.2$, which is the baseline value used in Mendoza [2010]. The moments are again similar across solution methods, and differ slightly more from the ones reported by Mendoza for the same reasons explained earlier. The only item that differs noticeably is the probability of Sudden Stops, which is about 2.0 percent in all the alternatives we solved here v. 3.3 percent in Mendoza's paper. We applied the same definition of Sudden Stops (i.e. coordinates $(b, k, s)$ in which the collateral constraint binds and the trade balance-GDP ratio is at least 2 percentage points above what the RBC model yields). The lower frequency of Sudden Stops is due to the approximately-continuous decision rules that we obtain using interpolation in our solutions v. decision rules forced to be on grid nodes in Mendoza's solution. This makes our estimates of the frequency with which $\mu(b, k, s)>0$, and of the trade balance adjustment implied by the associated $b^{\prime}(b, k, s)$ in those states, more accurate. In all of our results, the long-run probability of states with $\mu>0$ is about 2.6 percent, but 23 percent of these states do not yield a sufficiently large increase in the trade balance to classify as a Sudden Stop.

Euler equation errors are again very small and similar across solutions in Cols. (1)-(5), but now the FTI solution yields larger maximum and average absolute Euler equation errors. Still, this only results in means that differ to the first decimal, and makes very little difference in second- and higher-order moments. In terms of speed, the FiPIt method in Column (2) remains the fastest, and the ranking of the execution time of the other methods relative to FiPIt remains unchanged. All the speed ratios get closer to the execution time of the FiPIt solution, but the differences are still significant. In particular, FTI takes 2.5 times as long, and the two methods that use time iteration partially (by applying it only to the bond's Euler equation) take nearly 5 times as long. 
The difference in speed ratios narrows because in every state in which the constraint binds in each iteration, all the algorithms require a non-linear solver. FiPIt loses in relative terms because it still requires a higher number of iterations in order to converge (196) but now in each one it needs to solve a non-linear equation, while FTI still converges faster (in 94 iterations) and still solves two non-linear equations in each iteration. ${ }^{7}$

\footnotetext{
${ }^{7}$ This suggests that FiPIt can be again much faster than FTI in applications in which, as explained in Section 3, the structure of the occasionally binding constraint is such that FiPIt does not need a root-finder in states in which the constraint binds (e.g. $q_{t}^{b} b_{t+1} \geq-\kappa q_{t} k_{t+1}, q_{t}^{b} b_{t+1} \geq-\varphi$ ). We show results for a case like this in subsection 4.3 .
} 
Table 3: Long-run Moments \& Performance Metrics: Sudden Stops Model $(\kappa=0.2)$

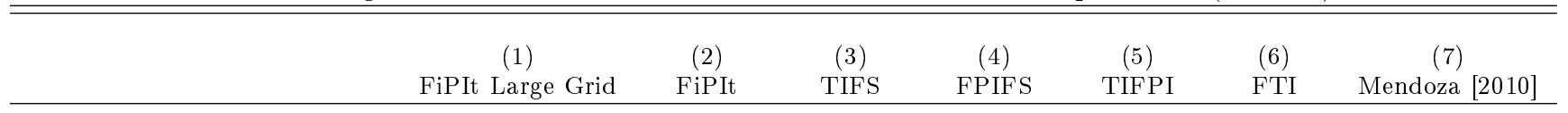

(a) Long-run moments

\begin{tabular}{|c|c|c|c|c|c|c|c|}
\hline \multicolumn{8}{|l|}{ Mean } \\
\hline$g d p$ & 393.629 & 393.619 & 393.626 & 393.618 & 393.626 & 393.549 & 388.339 \\
\hline$c$ & 273.910 & 274.123 & 274.074 & 274.124 & 274.073 & 274.011 & 267.857 \\
\hline$i$ & 67.482 & 67.481 & 67.484 & 67.481 & 67.484 & 67.459 & 65.802 \\
\hline$n x / g d p$ & 0.016 & 0.015 & 0.015 & 0.015 & 0.015 & 0.015 & 0.024 \\
\hline$k$ & 765.191 & 765.171 & 765.202 & 765.170 & 765.202 & 764.922 & 747.709 \\
\hline$b / g d p$ & 0.007 & 0.015 & 0.013 & 0.015 & 0.013 & 0.012 & -0.104 \\
\hline$q$ & 1.000 & 1.000 & 1.000 & 1.000 & 1.000 & 1.000 & 1.000 \\
\hline leverage ratio & -0.106 & -0.102 & -0.103 & -0.102 & -0.103 & -0.103 & -0.159 \\
\hline$v$ & 42.618 & 42.617 & 42.618 & 42.617 & 42.618 & 42.609 & 41.949 \\
\hline working capital & 76.660 & 76.658 & 76.659 & 76.658 & 76.659 & 76.644 & 75.455 \\
\hline \multicolumn{8}{|c|}{ Standard deviation (in percent) } \\
\hline$g d p$ & 3.91 & 3.94 & 3.94 & 3.94 & 3.94 & 3.94 & 3.85 \\
\hline$c$ & 3.95 & 4.03 & 4.02 & 4.03 & 4.02 & 4.03 & 3.69 \\
\hline$i$ & 13.33 & 13.33 & 13.33 & 13.33 & 13.33 & 13.32 & 13.45 \\
\hline$n x / g d p$ & 2.90 & 2.94 & 2.94 & 2.94 & 2.94 & 2.94 & 2.58 \\
\hline$k$ & 4.40 & 4.49 & 4.49 & 4.49 & 4.49 & 4.50 & 4.31 \\
\hline$b / g d p$ & 18.72 & 19.62 & 19.47 & 19.62 & 19.47 & 19.45 & 8.90 \\
\hline$q$ & 3.20 & 3.20 & 3.20 & 3.20 & 3.20 & 3.20 & 3.23 \\
\hline leverage ratio & 8.79 & 9.22 & 9.15 & 9.22 & 9.15 & 9.14 & 4.07 \\
\hline$v$ & 5.87 & 5.89 & 5.89 & 5.89 & 5.89 & 5.89 & 5.84 \\
\hline working capital & 4.33 & 4.35 & 4.35 & 4.35 & 4.35 & 4.36 & 4.26 \\
\hline \multicolumn{8}{|l|}{ Correlation with GDP } \\
\hline$g d p$ & 1.000 & 1.000 & 1.000 & 1.000 & 1.000 & 1.000 & 1.000 \\
\hline$c$ & 0.849 & 0.842 & 0.844 & 0.842 & 0.844 & 0.844 & 0.931 \\
\hline$i$ & 0.646 & 0.641 & 0.641 & 0.641 & 0.641 & 0.641 & 0.641 \\
\hline$n x / g d p$ & -0.122 & -0.117 & -0.118 & -0.117 & -0.118 & -0.120 & -0.184 \\
\hline$k$ & 0.757 & 0.761 & 0.761 & 0.761 & 0.761 & 0.761 & 0.744 \\
\hline$b / g d p$ & -0.133 & -0.120 & -0.119 & -0.120 & -0.119 & -0.117 & -0.298 \\
\hline$q$ & 0.400 & 0.387 & 0.387 & 0.387 & 0.387 & 0.387 & 0.406 \\
\hline leverage ratio & -0.125 & -0.111 & -0.111 & -0.111 & -0.111 & -0.108 & 0.258 \\
\hline$v$ & 0.831 & 0.832 & 0.832 & 0.832 & 0.832 & 0.832 & 0.823 \\
\hline working capital & 0.994 & 0.994 & 0.994 & 0.994 & 0.994 & 0.994 & 0.987 \\
\hline \multicolumn{8}{|c|}{ First-order autocorrelation } \\
\hline$g d p$ & 0.823 & 0.825 & 0.824 & 0.825 & 0.824 & 0.825 & 0.815 \\
\hline$c$ & 0.823 & 0.830 & 0.829 & 0.830 & 0.829 & 0.829 & 0.766 \\
\hline$i$ & 0.500 & 0.501 & 0.500 & 0.501 & 0.500 & 0.500 & 0.483 \\
\hline$n x / g d p$ & 0.589 & 0.601 & 0.598 & 0.601 & 0.598 & 0.598 & 0.447 \\
\hline$k$ & 0.964 & 0.962 & 0.962 & 0.962 & 0.962 & 0.962 & 0.963 \\
\hline$b / g d p$ & 0.989 & 0.990 & 0.990 & 0.990 & 0.990 & 0.990 & 0.087 \\
\hline$q$ & 0.444 & 0.447 & 0.446 & 0.447 & 0.446 & 0.446 & 0.428 \\
\hline leverage ratio & 0.991 & 0.992 & 0.992 & 0.992 & 0.992 & 0.992 & 0.040 \\
\hline$v$ & 0.776 & 0.777 & 0.777 & 0.777 & 0.777 & 0.777 & 0.764 \\
\hline working capital & 0.800 & 0.801 & 0.801 & 0.801 & 0.801 & 0.801 & 0.777 \\
\hline Prob. of Sudden Stops & $1.98 \%$ & $1.99 \%$ & $2.03 \%$ & $1.99 \%$ & $2.04 \%$ & $2.05 \%$ & $3.32 \%$ \\
\hline
\end{tabular}

(b) Performance metrics

Bonds Euler Equation

Max Log10 Abs. Euler Error

At Grid Points $(b, k, s)$

Mean Log10 Abs. Euler Error

$\begin{array}{ccccccc}-3.58 & -3.58 & -3.58 & -3.58 & -3.58 & -3.52 & - \\ (1,11,3) & (1,6,3) & (1,6,3) & (1,6,3) & (1,6,3) & (2,1,7) & - \\ -14.45 & -14.45 & -12.41 & -14.27 & -12.39 & -12.35 & - \\ & & & & & & \\ -15.38 & -15.37 & -15.42 & -15.37 & -15.42 & -4.06 & - \\ (72,1,7) & (72,1,7) & (32,1,7) & (72,1,7) & (32,1,7) & (1,1,7) & - \\ -16.22 & -16.22 & -16.07 & -16.04 & -16.21 & -12.45 & - \\ (72,60) & (72,30) & (72,30) & (72,30) & (72,30) & (72,30) & (80,60) \\ & & & & & & \\ 1985 & 810 & 3735 & 956 & 4136 & 1986 & - \\ 2.5 & 1.0 & 4.6 & 1.2 & 5.1 & 2.5 & - \\ 196 & 196 & 190 & 178 & 207 & 94 & -\end{array}$

Capital Euler Equation

Max Log10 Abs. Euler Error

At Grid Points $(b, k, s)$

Mean Log10 Abs. Euler Error

Grid size $(\# b, \# k)$

196

196

190

178

Number of iterations

Note: Column (1) and Co

Column (3) is for the TIFS methe are for the FiPIt algorithm, fixed-point iteration is used for both the bonds decision rule and the price of capital equation for the price of capital. Column (4) is for the FPIFS method, which uses fixed-point iteration for the bonds decision rule and the forward solution of the capital Euler equation for the price of capital. Column (5) is for the TIFPI method, which uses time iteration for the decision rule for bonds and fixed-point iteration for the price of capital. Column (6) is for the FTI method, which solves the bonds decision rule and the price of capital by solving the Euler equations for bonds and capital as two simultaneous non-linear equations. Sudden Stop states are defined as in Mendoza [2010]: states $(b, k, s)$ such that $\mu(b, k, s)>0$ and the trade balance-GDP ratio is at least 2 percentage points above its value in the RBC model. 
Since we found larger Euler equation errors in the SS model than in the RBC model, we also examined differences in the recursive equilibrium functions produced by each solution method relative to the FiPIt solution for the SS model. Table 4 shows the maximum and mean of the absolute value of the point-wise differences of the functions as a ratio of the corresponding FiPIt solution. The differences are generally negligible, except for the maximum differences for $b^{\prime}$ and $i$ in the FTI solution, which reach 9.94 and 2.25 respectively in states in which the corresponding denominator is very close to zero. Still, as shown in Table 3 this makes little difference in first moments and is nearly irrelevant for second- and higher-order moments.

Table 4: Absolute Values of Differences in Equilibrium Functions Relative to FiPIt Solution

Differences Relative to FiPIt Method

\begin{tabular}{lcccc} 
& $(1)$ & $(2)$ & $(3)$ & $(4)$ \\
& TIFS & FPIFS & TIFPI & FTI \\
\hline Max Difference & & & \\
$b^{\prime}$ & $3.17 \mathrm{e}+00$ & $5.71 \mathrm{e}-02$ & $3.21 \mathrm{e}+00$ & $9.94 \mathrm{e}+00$ \\
$k^{\prime}$ & $2.23 \mathrm{e}-04$ & $7.17 \mathrm{e}-07$ & $2.23 \mathrm{e}-04$ & $1.10 \mathrm{e}-02$ \\
$q$ & $6.04 \mathrm{e}-04$ & $1.99 \mathrm{e}-06$ & $6.04 \mathrm{e}-04$ & $1.30 \mathrm{e}-01$ \\
$c$ & $9.72 \mathrm{e}-05$ & $7.08 \mathrm{e}-07$ & $9.72 \mathrm{e}-05$ & $1.09 \mathrm{e}-02$ \\
$i$ & $8.75 \mathrm{e}-02$ & $5.79 \mathrm{e}-05$ & $8.74 \mathrm{e}-02$ & $2.25 \mathrm{e}+00$ \\
$L$ & $4.92 \mathrm{e}-05$ & $3.94 \mathrm{e}-07$ & $4.92 \mathrm{e}-05$ & $2.57 \mathrm{e}-03$ \\
$v$ & $9.08 \mathrm{e}-05$ & $7.27 \mathrm{e}-07$ & $9.08 \mathrm{e}-05$ & $4.76 \mathrm{e}-03$ \\
$g d p$ & $3.27 \mathrm{e}-05$ & $2.61 \mathrm{e}-07$ & $3.27 \mathrm{e}-05$ & $1.77 \mathrm{e}-03$ \\
& & & & \\
Mean & Difference & & & \\
$b^{\prime}$ & $6.29 \mathrm{e}-04$ & $1.10 \mathrm{e}-05$ & $6.37 \mathrm{e}-04$ & $4.35 \mathrm{e}-03$ \\
$k^{\prime}$ & $1.13 \mathrm{e}-05$ & $2.76 \mathrm{e}-07$ & $1.15 \mathrm{e}-05$ & $9.81 \mathrm{e}-05$ \\
$q$ & $3.16 \mathrm{e}-05$ & $7.61 \mathrm{e}-07$ & $3.22 \mathrm{e}-05$ & $5.64 \mathrm{e}-04$ \\
$c$ & $2.00 \mathrm{e}-05$ & $3.22 \mathrm{e}-07$ & $2.03 \mathrm{e}-05$ & $7.10 \mathrm{e}-05$ \\
$i$ & $1.74 \mathrm{e}-04$ & $3.27 \mathrm{e}-06$ & $1.76 \mathrm{e}-04$ & $1.51 \mathrm{e}-03$ \\
$L$ & $1.22 \mathrm{e}-06$ & $3.27 \mathrm{e}-09$ & $1.22 \mathrm{e}-06$ & $2.38 \mathrm{e}-05$ \\
$v$ & $2.24 \mathrm{e}-06$ & $6.03 \mathrm{e}-09$ & $2.25 \mathrm{e}-06$ & $4.40 \mathrm{e}-05$ \\
$g d p$ & $8.16 \mathrm{e}-07$ & $2.19 \mathrm{e}-09$ & $8.18 \mathrm{e}-07$ & $1.62 \mathrm{e}-05$ \\
\hline \hline
\end{tabular}

Figure 2 shows the ergodic marginal distributions of bonds and capital, and the ergodic joint marginal distribution of both variables produced by the FiPIt solution. As in the RBC case, the distributions are visually identical for all the other solution methods, including FTI. Comparing with the distributions of the RBC model shown in Figure 1, we find standard properties of SS models: The distribution of bonds shifts to the right because of the credit constraint and the strengthened 
precautionary saving incentives. The distribution of capital shows higher dispersion and a fatter left tail because of the fire-sales of capital in states in which the constraint binds.

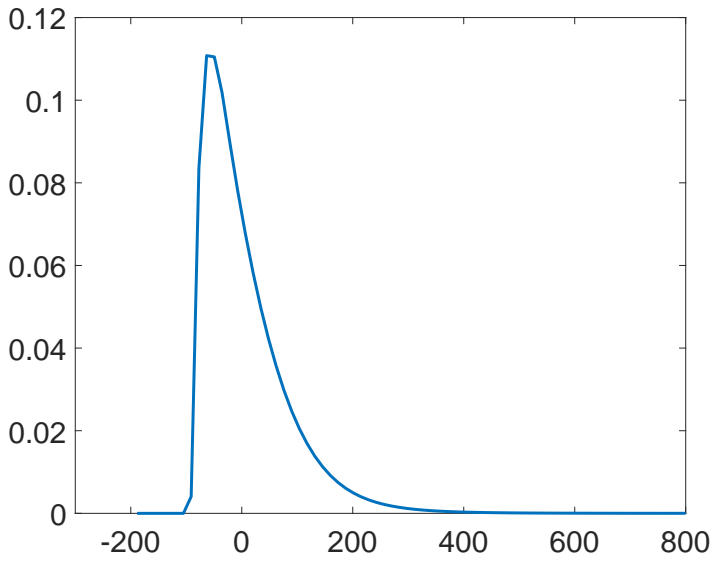

(a) Ergodic Bond Distribution

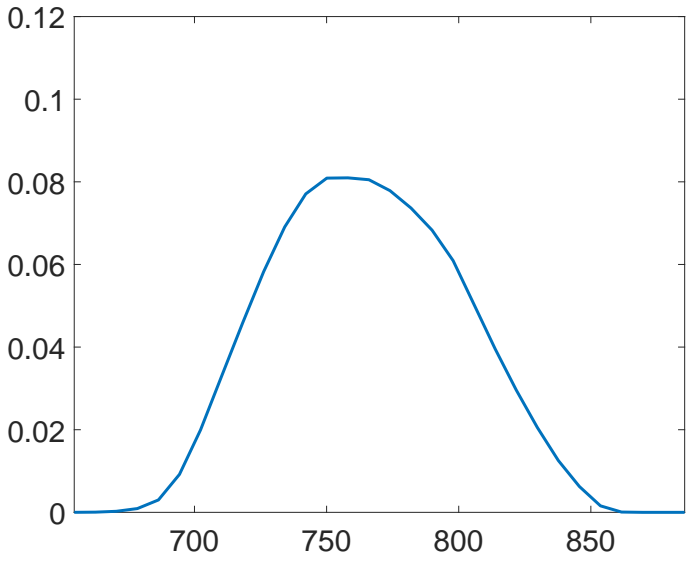

(b) Ergodic Capital Distribution

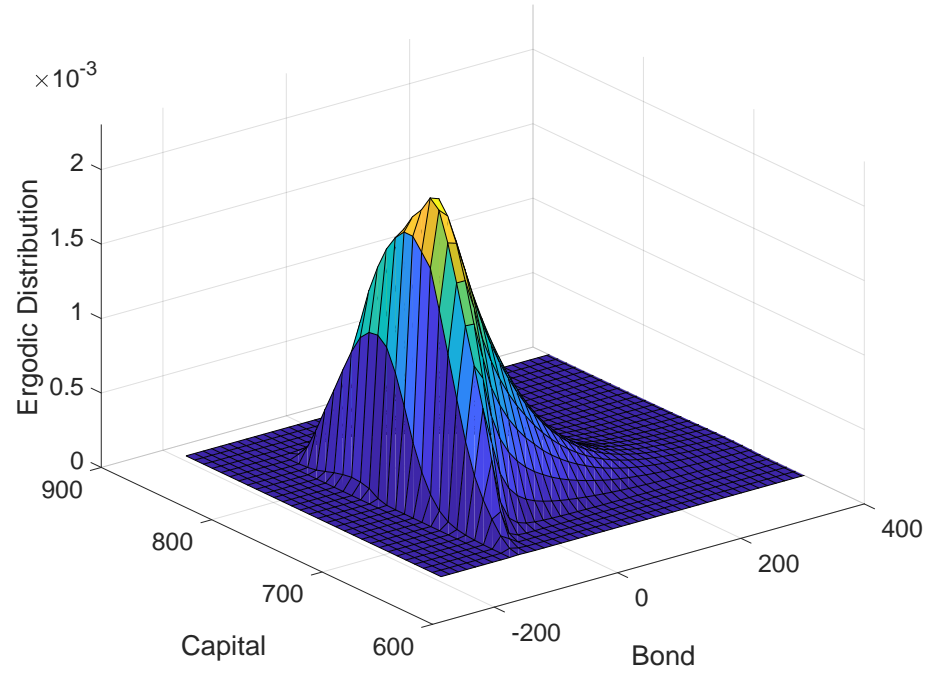

(c) Ergodic Distribution of Bonds and Capital

Figure 2: Long-run Distributions of the Sudden Stops Model solved with the FiPIt

Figure 3 shows the decision rules of bonds and capital, the pricing function of capital and the multiplier of the credit constraint for the RBC and SS models across the full state space of the endogenous states, $\mathbf{B} \otimes \mathbf{K}$, with $s$ evaluated at the adverse values of the model's three shocks (low TFP, high interest rate, high input prices). We show results obtained with the FiPIt method because the comparable plots for the other solution methods are visually identical. 
The four plots show significant differences between the RBC and SS solutions in the region where the credit constraint binds and near where it binds. In particular, the SS outcomes show significant non-linearities, whereas the RBC outcomes are approximately linear. The fire-sales of capital when the constraint binds, the resulting collapse in the price of capital, and the associated sharp reversal in the bond position as borrowing capacity collapses due to the capital fire-sales and the drop in the price of capital, are evident in plots (a) to (c). When the constraint binds, plot (d) shows that $\mu$ rises sharply and at an increasing rate as $b$ and/or $k$ fall. The sharp curvature of the recursive equilibrium functions of the SS model highlights the advantages of using a finite-state-space solution method, instead of a colocation method, as well as the importance of solving using first-order conditions and approximately-continuous decision rules. Decision rules that capture accurately the nonlinearities implied by occasionally binding constraints are critical for quantifying the positive and normative implications of this class of models. For their positive implications, the magnitude, dynamics and frequency of financial crises depends critically on the behavior of the decision rules near and at the constraint. For example, the magnitudes of the current account reversal and the collapse in investment depend on how far above (below) the 45 degree ray is the bonds (capital) decision rule in the region where the constraint binds. For the normative implications, quantifying the size of pecuniary externalities induced by the credit constraint and optimal macroprudential debt taxes that can prevent Sudden Stops hinges critically on how likely is the credit constraint expected to bind at $\mathrm{t}+1$ and how severely, conditional on being in a state in which it does not bind at $\mathrm{t}$ (see Bianchi and Mendoza [2018]). 


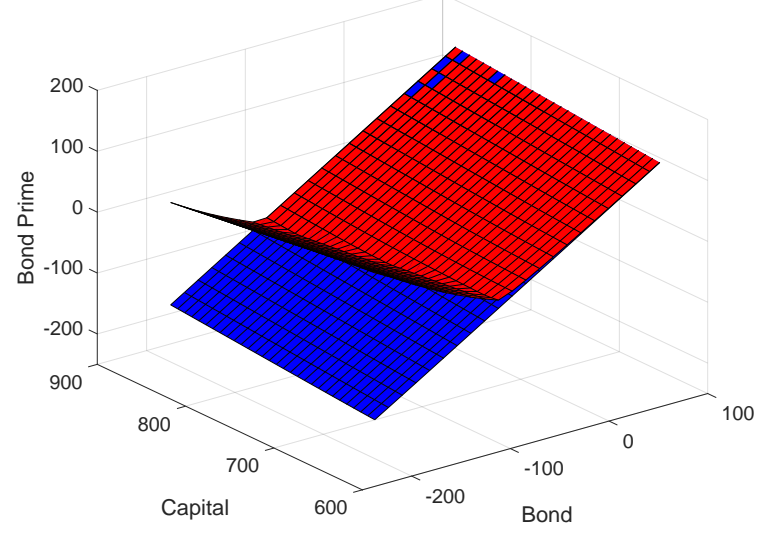

(a) Bonds decision rule

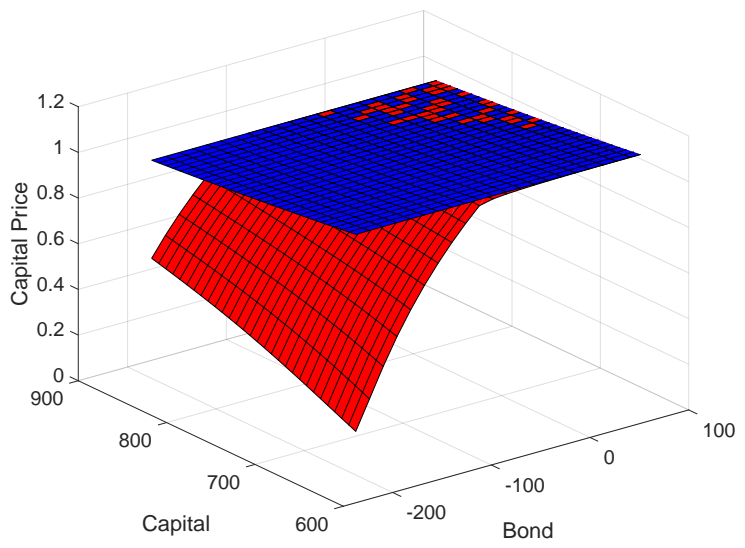

(c) Price of capital

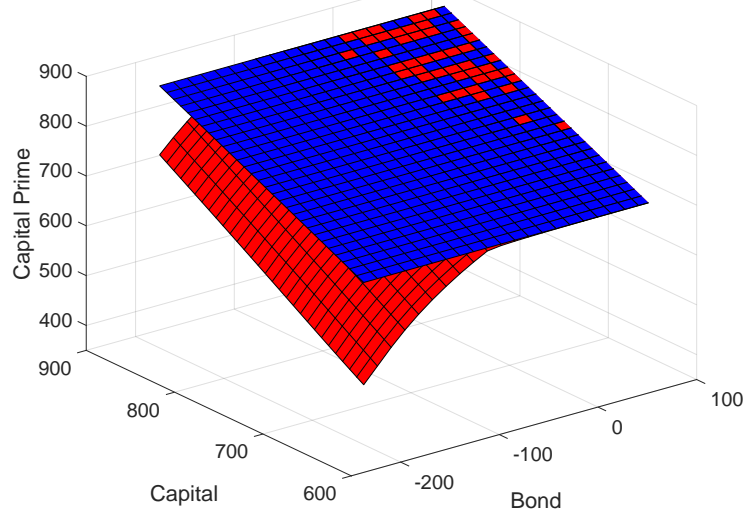

(b) Capital decision rule

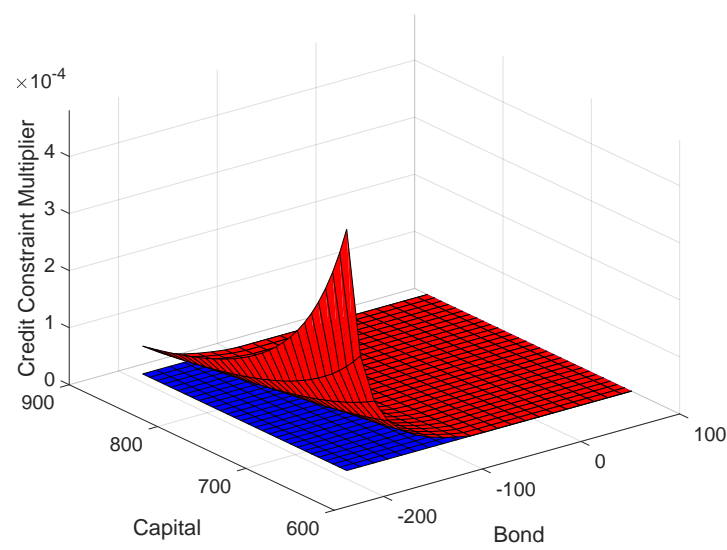

(d) Credit Constaint Multiplier

Figure 3: Decision Rules, Price of Capital and Credit Constraint Multiplier in Sudden Stops \& RBC Models

Note: All plots show solutions obtained with the FiPIt method. Surface plots in red (blue) are for the SS (RBC) model.

The decision rules suggest the presence of strong financial amplification and asymmetries in the SS model, but they do not control for whether particular points in $\mathbf{B} \otimes \mathbf{K}$ have positive probability in the stochastic steady state. States with zero-probability are irrelevant for economic fluctuations over the long run. To address this issue, we follow the methodology proposed by Mendoza [2010] to calculate long-run averages of impact amplification coefficients and report the results in Table 5. The coefficients in this Table measure the excess response of macro variables across the SS and RBC solutions for each triple $(b, k, s)$ that can be observed at any date $t$, separating the state space into Sudden Stop (SS) and non-Sudden Stop (NSS) regions. A triple $(b, k, s)$ belongs in the SS set 
if the trade balance-GDP ratio in the Sudden Stops model is 2 percentage points or more above its value in the RBC model at the same $(b, k, s)$, otherwise it belongs in the NSS region. The amplification coefficients for each variable at any state $(b, k, s)$ are calculated as differences relative to their values in the RBC model in the same state and expressed in percent of the unconditional mean of the variable also in the RBC model. ${ }^{8}$ The averages in the SS and NSS columns of the Table are computed using the limiting distribution of $(b, k, s)$ of the Sudden Stops model. Hence, the SS averages only reflect financial amplification in Sudden Stop events that can occur with positive probability in the stochastic stationary equilibrium. The results in the SS column by themselves measure financial amplification impact when a crisis occurs. Differences across the SS and NSS columns illustrate asymmetry, namely the amount by which shocks of identical magnitudes generate different effects when the collateral constraint is present and active v. when is not.

Table 5 shows results for the FiPIt and FTI solutions only, because the other methods yield nearly identical results. The coefficients produced by the FiPIt v. FTI solutions differ also very marginally, and in most instances they are the same up to the second decimal. The Table shows that the occasionally binding credit constraint induces significant amplification and asymmetry. Amplification coefficients on factor allocations and output are relatively smaller, because on impact at date-t when the credit constraint binds it can only affect them via its effect on working capital financing and hence on labor and imported inputs, since the date-t capital stock is pre-determined.

The results in Table 5 are qualitatively similar to those reported in Table 4 of Mendoza [2010], but quantitatively there are significant differences, which are again due to differences in model structure (i.e. endogenous v. exogenous discounting) and solution methods (i.e. decision rules forced to be on grid nodes v. interpolated decision rules, value function iteration v. first-order conditions). The SS coefficients of "supply side" variables (i.e. GDP, labor, imported inputs and working capital) are smaller in our solution, while those for the rest of the variables (particularly investment and the price of capital) are larger. Moreover, for supply-side variables in the NSS region we obtained near-zero amplification while Mendoza obtained figures ranging between -0.11 and -0.29. This is again reflecting the improved accuracy of our algorithm. In theory, since $k$ is pre-determined at each date $t$ and preferences assume away the wealth effect on labor supply,

\footnotetext{
${ }^{8}$ For variables that are defined as ratios, the coefficient is simply the difference in the Sudden Stops model relative to the RBC model.
} 
when $\mu(b, k, s)=0$ the set of optimality conditions is the same in the RBC and SS models and in both cases all supply-side variables depend only on $(k, s)$. Thus, amplification coefficients for these variables should indeed be very close to zero numerically. The coefficients around -0.11 to -0.29 Mendoza obtained for these variables in NSS states result from approximation errors when averaging outcomes for states in which the NSS and SS regions are adjacent, and in determining the value of $\mu(b, k, s)$ when assigning $(b, k, s)$ triples to the SS and NSS sets.

Table 5: Amplification and Asymmetry of Sudden Stop events

\begin{tabular}{lcccc}
\hline \hline & \multicolumn{2}{c}{$(1)$} & \multicolumn{2}{c}{$(2)$} \\
& FiPIt & \multicolumn{2}{c}{ FTI } \\
& SS & NSS & SS & NSS \\
\hline$g d p$ & -0.777 & -0.001 & -0.789 & -0.001 \\
$c$ & -3.849 & -0.255 & -3.882 & -0.260 \\
$i$ & -24.965 & -1.036 & -25.384 & -1.089 \\
$q$ & -6.090 & -0.253 & -6.194 & -0.266 \\
$n x / g d p$ & 4.033 & 0.233 & 4.047 & 0.238 \\
$b^{\prime} / g d p$ & 4.215 & 0.251 & 4.229 & 0.257 \\
$k^{\prime} / g d p$ & -1.667 & -0.105 & -1.680 & -0.110 \\
$l e v$. ratio & 1.166 & 0.081 & 1.167 & 0.082 \\
$L$ & -1.178 & -0.001 & -1.196 & -0.002 \\
$v$ & -2.146 & -0.003 & -2.180 & -0.003 \\
$w$. cap & -2.160 & -0.003 & -2.193 & -0.003 \\
\hline
\end{tabular}

Note: Sudden Stop (SS) states are defined as states in which the collateral constraint binds and the trade balance-GDP ratio in the Sudden Stop model is more than 2 percentage points above the trade balance-GDP ratio of the $\mathrm{RBC}$ model. The coefficients are computed as mean differences relative to the $\mathrm{RBC}$ model in percent of the $\mathrm{RBC}$ unconditional averages.

Figure 4 shows the results of a Sudden Stops event analysis applying the same methodology as in Mendoza [2010]. We run a 100,000 period time-series simulation of the model and define Sudden Stop events as periods in which the collateral constraint binds, the trade balance-GDP ratio is one standard deviation above its HP trend and GDP is one standard deviation below its HP trend. We show results based on the FiPIt solution. The event plots produced by the other methods are very similar and hence we do not include them in the paper. 
Figure 4: Sudden Stop Event Windows (means of deviations from HP trend)
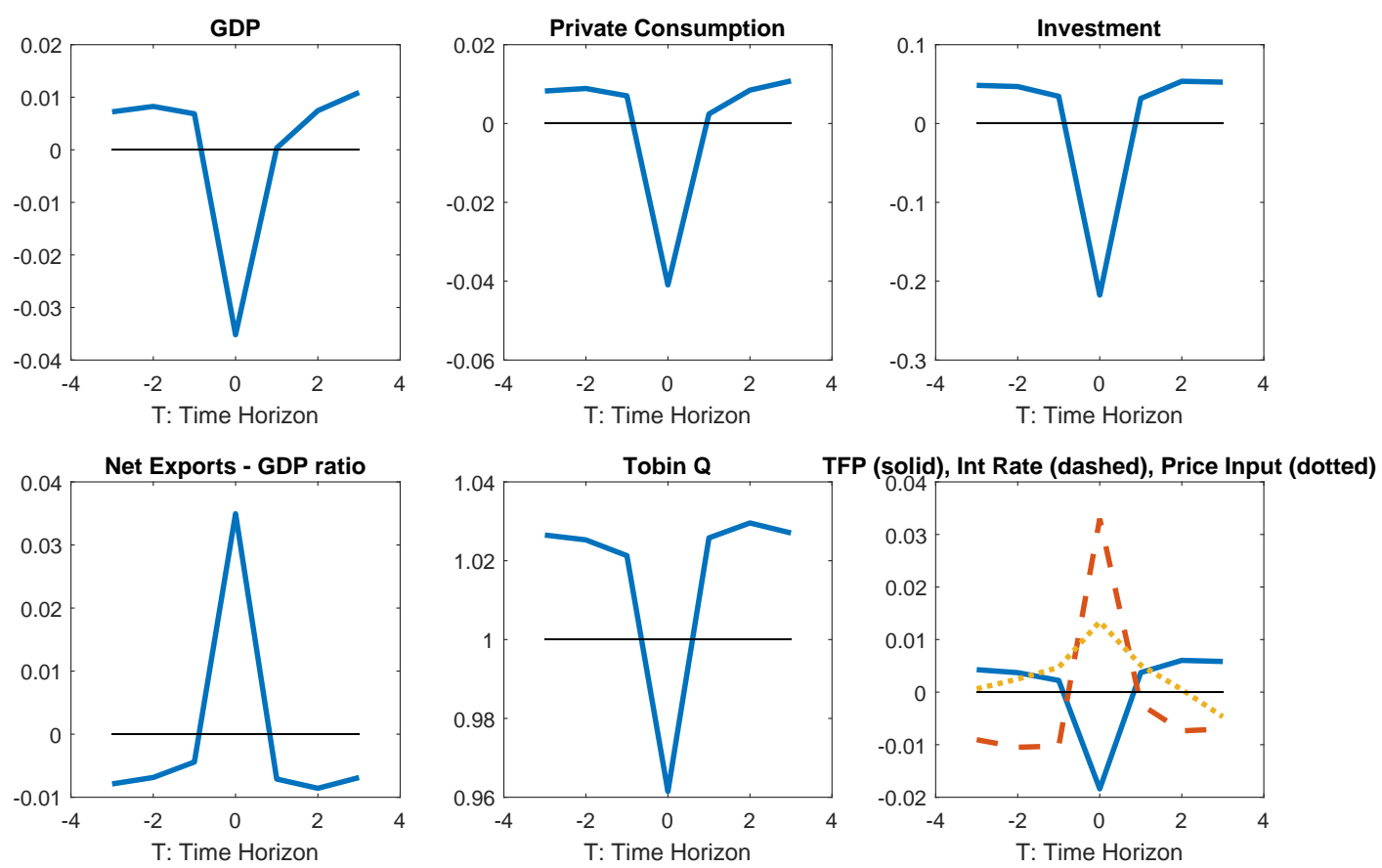

Note: Sudden Stop events in the model simulations are defined as events in which the collateral constraint binds, the trade balance-GDP ratio is one standard deviation above its HP trend and GDP is one standard deviation below its HP trend. Tobin's Q is shown in levels.

\subsection{Robustness Analysis \& Credit Constraint Variations}

The last set of experiments evaluates the robustness and stability of the FiPIt algorithm by examining its performance relative to the time iteration method for various parameter changes applied to the SS model. This is important in light of the potential instability of fixed-point iteration methods. As documented below, the FiPIt method remains stable and continues to outperform the FTI method in all the experiments. We also examine results for variations of the formulation of the credit constraint for which FiPIt does not require using a non-linear solver in states in which the constraint binds and found even larger gains in execution time.

Tables 6,7 and 8 show long-run moments and performance metrics obtained by solving the Sudden Stops model using the FiPIt and FTI methods for these parameter changes: (a) removing working capital $(\phi=0)$; (b) lowering the discount factor $(\beta=0.91)$; (c) reducing the collateral coefficient $(\kappa=0.15)$; (d) increasing the collateral coefficient $(\kappa=0.25)$; (e) increasing the labor disutility coefficient $(\omega=2.5)$; and (f) increasing the relative risk aversion coefficient $(\sigma=3.0)$. For 
each parameter variation, the grids of capital and bonds were re-sized to obtain the fastest solution that does not distort the quantitative results, using identical grids for the FiPIt and FTI solutions. Still, this resulted in grids of about the same dimensions: 71 or 72 nodes in $\mathbf{B}$ and 30 nodes in $\mathbf{K}$, except for case (e) that needed only 62 nodes in $\mathbf{B}$.

The dominance of the FiPIt method is robust to all these parameter changes, and in all cases the algorithm is stable and yields solutions nearly identical to the FTI results. Comparing across the cases in which the root-finder is needed to solve allocations when the credit constraint binds (i.e. excluding case (a)), FTI is 2.0 to 6.0 times slower than FiPIt depending on which scenario is considered. Comparing v. the scenario in which FiPIt does not need the root finder when the constraint binds (Col. (2) of Table 6), FTI is 13.0 times slower. Moreover, in most cases FiPIt did not require changing the values of the dampening parameters for the updates of the decision rule for bonds $\left(\rho^{b}=1\right)$, the credit constraint multiplier $\left(\rho^{\mu}=1\right)$ and the pricing function $\left(\rho^{q}=0.3\right)$.

It is worth noting that the time iteration solutions required about the same number of iterations (between 87 and 100) and execution time in all the experiments except case (e), which has the smaller $\mathbf{B}$ grid and used about the same number of iterations but solved faster than the other timeiteration solutions. There is more variation in both number of iterations and execution times in the FiPIt solutions, but the two tend to move together: The slowest solution was for case (b) which took 1,130 seconds and 244 iterations.

For the case without working capital (case (a)), Column (2) shows the results that FiPIt yields when the code is modified to take into account that, since the credit constraint is now of the form $B_{j+1}(b, k, s) / R \geq-\kappa \hat{q}_{j}(b, k, s) K_{j}(b, k, s)$, a root-finder is not needed to solve when the constraint binds, as explained in Section 3. We also solved an additional experiment with an alternative credit constraint in the same class that does not require a non-linear solver: $B_{j+1}(b, k, s) / R \geq \varphi$ with $\varphi$ set one standard deviation below the average of $b^{\prime}$ in the limiting distribution of the RBC model. These experiments illustrate the large additional gain in speed that FiPIt yields when used to solve models with constraints like these. In Case (a), the FiPIt solution is obtained in almost one-third of the time taken by the FiPIt algorithm that uses the non-linear solver, which implies that FiPIt is faster than the time iteration solution by a factor of 13.0 (v. 5.6 with the FiPIt algorithm that uses the non-linear solver). In the case with the constraint given by $\varphi$, the FiPIt solution is faster than the time iteration method by a factor of 17.9 . 
Table 6: Sudden Stops Model Variations: Working Capital \& Discounting

\begin{tabular}{|c|c|c|c|}
\hline \multirow{5}{*}{\multicolumn{2}{|c|}{ (a) Working Capital $\phi=0$}} & \multirow{2}{*}{\multicolumn{2}{|c|}{ (b) Discount factor $\beta=0.91$}} \\
\hline & & & \\
\hline & & & \\
\hline & & & \\
\hline & & & \\
\hline & & & \\
\hline & & & \\
\hline & $\begin{array}{c}(3) \\
\text { FTI }\end{array}$ & $\begin{array}{c}\text { (b) Disc } \\
(1) \\
\text { FiPIt }\end{array}$ & $\begin{array}{l}\text { r } \beta=0.91 \\
(3) \\
\text { FTI }\end{array}$ \\
\hline
\end{tabular}

(a) Long-run moments

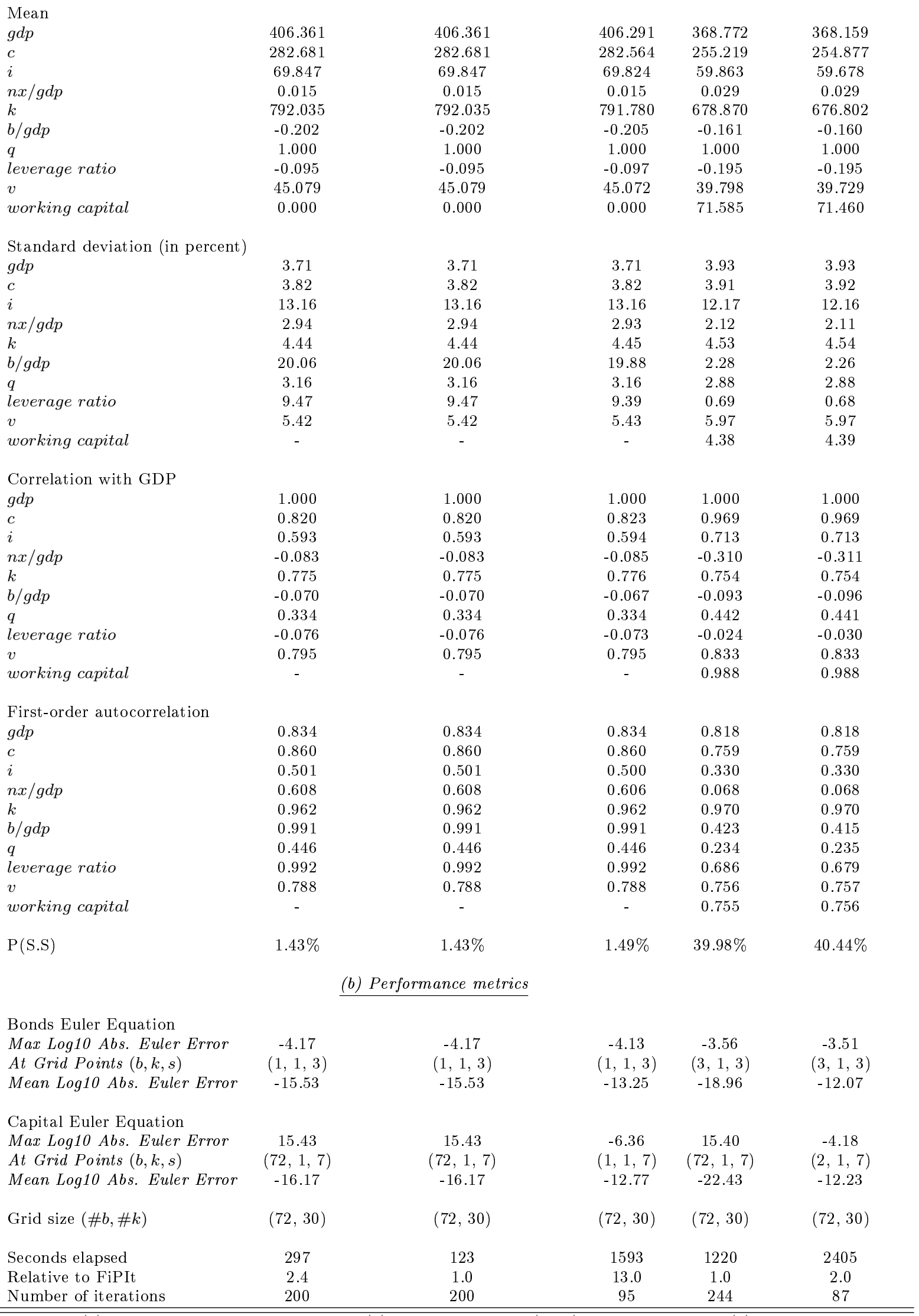

Note: Columns (1) are FiPIt solutions and Columns (3) are time iteration (FTI) solutions. Column (2) shows results for the model with $\phi=0$ obtained with the FiPIt algorithm without using a non-linear solver when $\mu>0$, since it is not needed. 
Table 7: Sudden Stops Model Variations: Collateral Coefficient

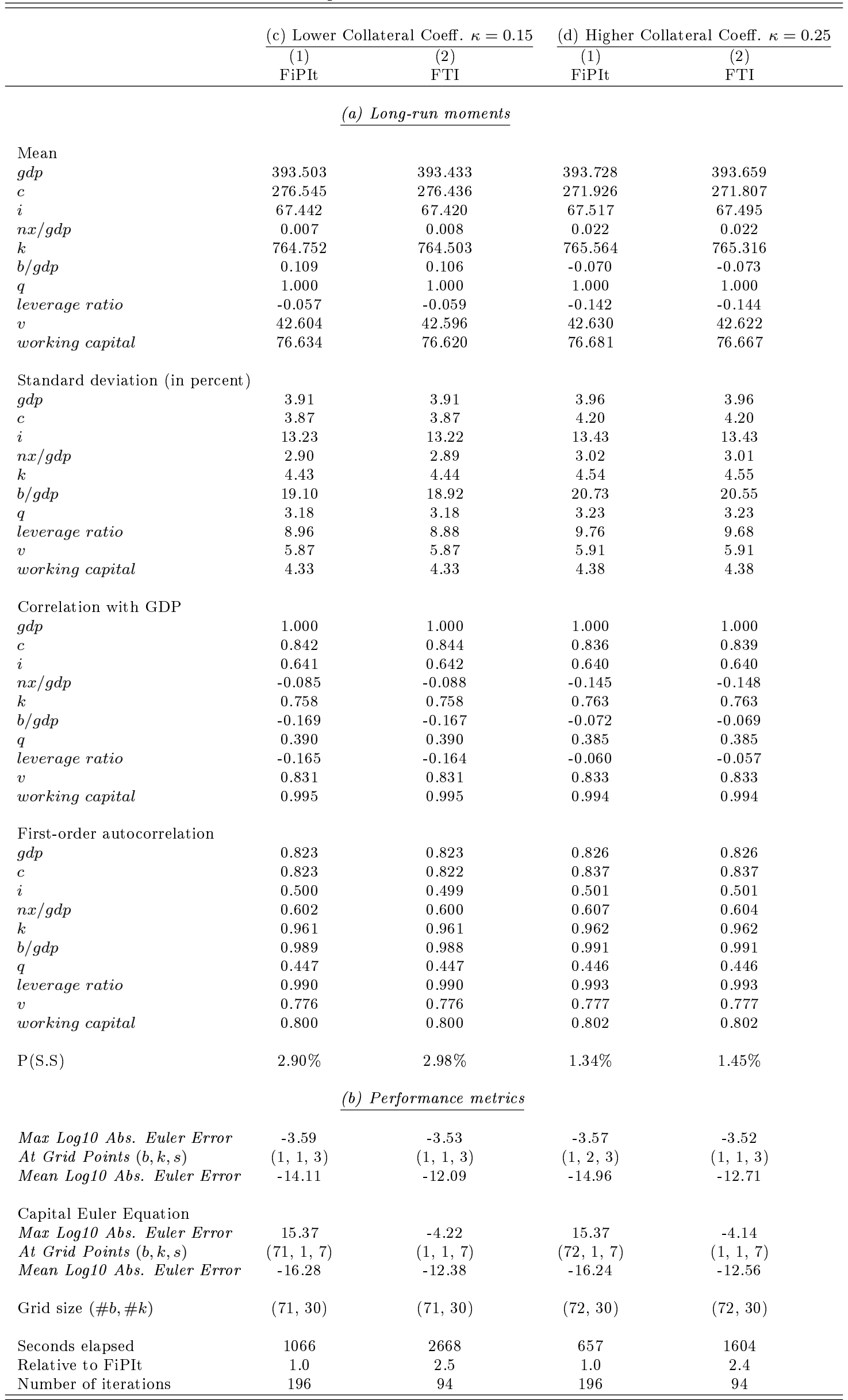

Note: Columns (1) are for the FiPIt algorithm. Columns (2) are for the full time iteration method (FTI). 
Table 8: Sudden Stops Model Variations: Labor Elasticity \& Risk Aversion

\begin{tabular}{ccccc}
\hline \hline & \multicolumn{2}{c}{ (e) Higer Labor Coef. $\omega=2.5$} & & (f) Higher Risk Aversion $\sigma=3$ \\
\cline { 2 - 3 }$(1)$ & $(2)$ & & $(1)$ & $(2)$ \\
FiPIt & FTI & & FiPIt & FTI \\
\hline
\end{tabular}

(a) Long-run moments

\begin{tabular}{|c|c|c|c|c|}
\hline \multicolumn{5}{|l|}{ Mean } \\
\hline$g d p$ & 110.477 & 110.441 & 393.797 & 393.722 \\
\hline$c$ & 78.995 & 78.897 & 277.504 & 277.357 \\
\hline$i$ & 18.937 & 18.924 & 67.534 & 67.509 \\
\hline$n x / g d p$ & -0.007 & -0.006 & 0.005 & 0.005 \\
\hline$k$ & 214.735 & 214.586 & 765.801 & 765.531 \\
\hline$b / g d p$ & 0.289 & 0.278 & 0.138 & 0.134 \\
\hline$q$ & 1.000 & 1.000 & 1.000 & 1.000 \\
\hline leverage ratio & 0.027 & 0.023 & -0.044 & -0.045 \\
\hline$v$ & 11.960 & 11.956 & 42.639 & 42.630 \\
\hline working capital & 21.514 & 21.507 & 76.697 & 76.682 \\
\hline \multicolumn{5}{|c|}{ Standard deviation (in percent) } \\
\hline$g d p$ & 3.33 & 3.33 & 3.97 & 3.98 \\
\hline$c$ & 6.31 & 6.24 & 4.35 & 4.35 \\
\hline$i$ & 13.11 & 13.11 & 13.46 & 13.46 \\
\hline$n x / g d p$ & 5.39 & 5.31 & 3.25 & 3.24 \\
\hline$k$ & 4.32 & 4.34 & 4.59 & 4.61 \\
\hline$b / g d p$ & 56.17 & 55.03 & 28.69 & 28.55 \\
\hline$q$ & 3.17 & 3.17 & 3.23 & 3.23 \\
\hline leverage ratio & 26.48 & 25.94 & 13.55 & 13.49 \\
\hline$v$ & 5.40 & 5.41 & 5.91 & 5.92 \\
\hline working capital & 3.76 & 3.77 & 4.39 & 4.40 \\
\hline \multicolumn{5}{|c|}{ Correlation with GDP } \\
\hline$g d p$ & 1.000 & 1.000 & 1.000 & 1.000 \\
\hline$c$ & 0.451 & 0.463 & 0.744 & 0.747 \\
\hline$i$ & 0.614 & 0.614 & 0.638 & 0.639 \\
\hline$n x / g d p$ & -0.077 & -0.084 & -0.059 & -0.062 \\
\hline$k$ & 0.755 & 0.756 & 0.765 & 0.765 \\
\hline$b / g d p$ & -0.039 & -0.034 & -0.058 & -0.054 \\
\hline$q$ & 0.366 & 0.365 & 0.381 & 0.381 \\
\hline leverage ratio & -0.043 & -0.037 & -0.048 & -0.044 \\
\hline$v$ & 0.797 & 0.797 & 0.834 & 0.834 \\
\hline working capital & 0.992 & 0.992 & 0.995 & 0.995 \\
\hline \multicolumn{5}{|c|}{ First-order autocorrelation } \\
\hline$g d p$ & 0.820 & 0.821 & 0.828 & 0.828 \\
\hline$c$ & 0.941 & 0.939 & 0.894 & 0.894 \\
\hline$i$ & 0.489 & 0.489 & 0.506 & 0.506 \\
\hline$n x / g d p$ & 0.857 & 0.852 & 0.733 & 0.731 \\
\hline$k$ & 0.960 & 0.960 & 0.963 & 0.963 \\
\hline$b / g d p$ & 0.995 & 0.995 & 0.995 & 0.995 \\
\hline$q$ & 0.435 & 0.435 & 0.450 & 0.450 \\
\hline leverage ratio & 0.995 & 0.995 & 0.996 & 0.996 \\
\hline$v$ & 0.768 & 0.769 & 0.779 & 0.779 \\
\hline working capital & 0.793 & 0.794 & 0.804 & 0.805 \\
\hline $\mathrm{P}(\mathrm{S} . \mathrm{S})$ & $3.38 \%$ & $3.66 \%$ & $1.29 \%$ & $1.35 \%$ \\
\hline
\end{tabular}

(b) Performance metrics

Bonds Euler Equation

Max Log10 Abs. Euler Error

At Grid Points $(b, k, s)$

Mean Log10 Abs. Euler Error

$-3.43$

$-3.37$

$-3.82$

$-3.75$

$(1,1,7)$

$(1,1,7)$

$(2,1,7)$

$(2,1,7)$

$-14.27$

$-13.16$

$-15.11$

$-12.41$

Capital Euler Equation

Max Log10 Abs. Euler Error

At Grid Points $(b, k, s)$

14.09

$(62,1,7)$

$-4.46$

16.30

$-3.90$

Mean Log10 Abs. Euler Error

$-14.76$

$-12.52$

$(71,1,7)$

$(1,1,7)$

Grid size $(\# b, \# k)$

$(62,30)$

$(62,30)$

$(71,30)$

$(71,30)$

Seconds elapsed

$\begin{array}{llll}283 & 1700 & 1108 & 3533\end{array}$

Relative to FiPIt

$1.0 \quad 6.0$

$\begin{array}{cc}1108 & 3533 \\ 1.0 & 3.2\end{array}$

Number of iterations

$98-246$

100

Note: Columns (1) are for the FiPIt algorithm. Columns (2) are for the full time iteration method (FTI). 


\subsection{Sketch of Other FiPIt Applications}

Applying the FiPIt algorithm requires mainly a fixed-point iteration strategy to iterate on recursive functions using Euler equations. In the RBC and SS models we solved, we examined, FiPIt was applied to the Euler equation for bonds to solve for the bonds decision rule and to the Euler equation for capital to solve for the price of capital. The Tobin's Q investment optimality condition was used to determine the decision rule for capital given the capital pricing function. It is possible to rearrange the solution in other ways that FiPIt may still accommodate, for example conjecturing the bonds and capital decision rules and using the two Euler equations to solve for their updates.

Applying the above principles to other model specifications so that they can be solved using FiPIt seems relatively straightforward. We provide here a brief sketch of four examples:

1. Mendoza [1995]: This is an RBC small open economy with incomplete markets and three sectors, quadratic capital adjustment costs given by $(\phi / 2)\left(k_{t+1}-k_{t}\right)^{2}$, and a maximum debt limit as the only occasionally binding constraint. The model has endogenous discounting, but consider a variant with a standard constant discount factor. The adjustment costs formulation does not satisfy the Hayashi conditions required for the average and marginal Tobin's Q to be the same, but for implementing FiPIt define a quasi capital pricing function given by $q_{t} \equiv 1+\phi\left(k_{t+1}-k_{t}\right)$, so that given a conjecture of this pricing function we can obtain an implied capital decision rule. Start with this pricing conjecture and a conjectured bonds decision rule. The model's equilibrium conditions, the implied capital decision rule, and the bonds decision rule can be used so that the resource constraint for tradable goods yields an implied decision rule for tradables consumption. FiPIt can then be used on the Euler equation for bonds to solve for a new tradables consumption decision rule, and the resource constraint yields a new bonds decision rule. FiPIt can then be applied to the Euler equation for capital to solve for a new $q$ function.

2. Ludwig and Schön [2018]: This is a model of optimal human capital accumulation $h$ with a no-borrowing constraint on an asset $a$ that pays an exogenous interest rate $R$ (i.e. a small open economy). Human capital depreciates at rate $\delta$ and is produced with a concave function of human capital investment $f(i)$. Agents have CRRA period utility and an exogenous probability of survival given by an increasing, concave function $s(h)$. To solve using FiPIt, start 
with a conjectured decision rule for assets $\hat{A}(a, h)$ and a conjecture for the shadow relative price of human capital investment $\hat{\tilde{\mu}}(a, h)$ where $\mu \equiv \mu / \lambda$ and $\lambda$ and $\mu$ are the multipliers on the resource constraint and law of motion of human capital accumulation respectively. Given these conjectures, the model's equilibrium conditions yield implied decision rules for human capital, consumption and investment in human capital. Then FiPIt can be applied to the Euler equation on assets to solve for a new consumption decision rule and using the result in the resource constraint yields a new decision rule for assets $A(a, h)$. If $A(a, h)<0$, re-define the decision rule as $A(a, h)=0$, set the associated consumption to the amount supported by the resource constraint, and compute the ratio $\psi / \lambda$ (where $\psi$ is the multiplier on the noborrowing constraint). Finally, rewrite the Euler equation for human capital in terms of the ratio $\mu / \lambda$ and apply FiPIt to solve for a new decision rule for $\tilde{\mu}$. There is no need to use a root-finder in this case.

3. Mendoza and Smith [2006]: This is a stochastic model of a small open economy in which agents trade in world bond markets and in a market where equity on the economy's capital can be bought by foreign investors, who face a quadratic cost of purchasing equity. A productivity shock affects equity returns. There is a credit constraint imposing a limit on the ratio of debt to the market value of the equity holdings of domestic agents, and a short-selling limit on the equity position. Given conjectures of the decision rule for bonds and the equity pricing function, the optimality condition of foreign investors and market clearing conditions yield an implied decision rule for equity holdings (the quadratic adjustment cost plays a role similar to the capital adjustment cost in the SS model we solved earlier). Given these, the resource constraint of the small open economy yields a decision rule for consumption. Assuming the credit constraints do not bind, FiPIt can then be applied to the bonds Euler equation to solve for a new consumption decision rule, and the resource constraint yields a new bonds decision rule. If the latter yields a value that violates the credit constraint, the constraint is imposed with equality to obtain new values for the bonds decision rule and consumption, and for the ratio of the multiplier of the borrowing constraint. Finally, FiPIt is applied to the Euler equation for equity holdings to obtain a new equity pricing function.

4. Huggett [1993]: This is one of the canonical heterogeneous agents models in which a continuum 
of agents trade non-state-contingent debt facing idiosyncratic Markov income shocks and a maximum debt limit. The optimization problem solved by an individual agent, who takes an exogenously-determined value of the interest rate as given, is identical to that of the small open endowment economy studied in Section 2, which has only one endogenous state variable. Start with a conjectured decision rule for bonds, use the resource constraint to obtain the implied decision rule for consumption. Then apply FiPIt to solve for a new consumption decision rule, and use the resource constraint to obtain a new bonds decision rule. If the latter violates the maximum debt limit, redefine to bonds decision rule to match the debt limit and set the associated consumption decision rule to the amount supported by the resource constraint. Iterate to convergence on the bonds decision rule and then use the decision rules and Markov process of income shocks to compute the ergodic distribution of bonds and income (i.e. the wealth and income distribution across agents). The difference with the small open economy is that now the interest rate is also part of the solution. The ergodic distribution is used to compute the aggregate demand for bonds (i.e. the mean of asset demand across agents), which must be equal zero at equilibrium in order to clear the bond market. If it yields exceeds demand (supply), the interest rate is reduced (increased) until the market-clearing condition holds up to a convergence criterion. Again FiPIt does not require a root finder.

\section{Conclusions}

FiPIt is a simple and fast algorithm designed to solve macroeconomic models with two endogenous state variables and occasionally binding constraints using widely used software. The algorithm applies fixed-point iteration on the Euler equations and by doing so it avoids solving the Euler equations as a non-linear system, as with the standard time iteration method, and does not require interpolation of decision rules over irregular grids, as with the endogenous grids method. Analytic solutions are obtained for recursive equilibrium functions in each iteration of the algorithm, and standard bi-linear interpolation for obtaining these analytic solutions remains applicable.

The FiPIt algorithm can handle a large class of occasionally binding constraints, including constraints set to fixed values as well as constraints that depend on endogenous variables. If the constraints are such that equilibrium allocations and prices when the constraints bind must be 
solved jointly with their associated multipliers, FiPIt does need a root-finder in states in which the constraint bind, but for a large class of constraints the two can be solved separately and FiPIt does not require a non-linear solver anywhere. In contrast, the endogenous grid method requires a root finder whenever the constraint binds.

We documented the performance gains and accuracy of FiPIt by comparing the solutions it produces for RBC and Sudden Stops models of a small open economy vis-a-vis solutions obtained with the time iteration method, and hybrid methods that combine fixed-point and time iteration techniques. In addition, we explored the robustness of our algorithm by documenting solutions for six parameter variations. The algorithm was coded in Matlab and executed in a standard Windows laptop. In all cases, FiPIt produced quantitative outcomes nearly identical to time iteration results at large gains in speed and comparable accuracy as measured by Euler equation errors. Time iteration solutions exceeded execution time relative to FiPIt solutions by factors of 2.0 to 17.9. The largest gains were obtained in cases in which FiPIt does not use root-finders anywhere, which include the RBC solution (since it only has a standard maximum debt constraint) and instances of the Sudden Stops model in which solving for allocations when the credit constraint binds does not require a non-linear solver. In these cases, time iteration took 15.1 and 17.9 times longer than FiPIt. In the baseline Sudden Stops model, which does need the solver to determine allocations when the constraint binds, time iteration took 2.5 times longer than FiPIt to solve the model.

The FiPIt algorithm can be extended to other models with two endogenous states. Performance gains are likely to be even larger if the algorithm is coded in languages that are more efficient at handling high-dimensional, sequential loops and parallel optimization, such as Julia, Fortran or Python. The large gains in speed and simplicity of the algorithm also open up the possibility of exploring research topics such as Bayesian estimation of models of financial crisis driven by occasionally binding collateral constraints. 


\section{References}

S Borağan Aruoba, Jesus Fernandez-Villaverde, and Juan F Rubio-Ramirez. Comparing solution methods for dynamic equilibrium economies. Journal of Economic dynamics and Control, 30(12): 2477-2508, 2006.

Javier Bianchi and Enrique G. Mendoza. Optimal time-consistent macroprudential policy. Journal of Political Economy, 126(2):588-634, 2018.

Javier Bianchi, Chenxin Liu, and Enrique G Mendoza. Fundamentals news, global liquidity and macroprudential policy. Journal of International Economics, 99:S2-S15, 2016.

Emine Boz and Enrique G Mendoza. Financial innovation, the discovery of risk, and the us credit crisis. Journal of Monetary Economics, 62:1-22, 2014.

Johannes Brumm and Michael Grill. Computing equilibria in dynamic models with occasionally binding constraints. Journal of Economic Dynamics and Control, 38:142-160, 2014.

Christopher D Carroll. The method of endogenous gridpoints for solving dynamic stochastic optimization problems. Economics letters, 91(3):312-320, 2006.

Christopher D Carroll. Lecture notes on solution methods for representative agent dynamic stochastic optimization problems. Mimeo, 2011.

Wilbur John Coleman. Solving the stochastic growth model by policy-function iteration. Journal of Business 63 Economic Statistics, 8(1):27-29, 1990.

Bora Durdu, Oliver de Groot, and Enrique Mendoza. Global v. local methods in the quantitative analysis of open-economy models with incomplete markets. Mimeo, 2019.

Jeremy Greenwood, Zvi Hercowitz, and Gregory W Huffman. Investment, capacity utilization, and the real business cycle. The American Economic Review, pages 402-417, 1988.

Luca Guerrieri and Matteo Iacoviello. Occbin: A toolkit for solving dynamic models with occasionally binding constraints easily. Journal of Monetary Economics, 70:22-38, 2015. 
Mark Huggett. The risk-free rate in heterogeneous-agent incomplete-insurance economies. Journal of economic Dynamics and Control, 17(5-6):953-969, 1993.

Kenneth L Judd. Numerical methods in economics. MIT press, 1998.

Lars Ljungqvist and Thomas J Sargent. Recursive macroeconomic theory. MIT press, 2012.

Alexander Ludwig and Matthias Schön. Endogenous grids in higher dimensions: Delaunay interpolation and hybrid methods. Computational Economics, 51(3):463-492, 2018.

Enrique G Mendoza. The terms of trade, the real exchange rate, and economic fluctuations. International Economic Review, pages 101-137, 1995.

Enrique G Mendoza. Sudden stops, financial crises, and leverage. American Economic Review, 100 (5):1941-66, 2010.

Enrique G Mendoza and Katherine A Smith. Quantitative implications of a debt-deflation theory of sudden stops and asset prices. Journal of International Economics, 70(1):82-114, 2006.

Pontus Rendahl. Inequality constraints and euler equation-based solution methods. The Economic Journal, 125(585):1110-1135, 2015. 